\title{
Protein Denaturation, Zero Entropy Temperature, and the Structure of Water around Hydrophobic and Amphiphilic Solutes
}

\author{
Kazimieras Tamoliūnas ${ }^{\mathrm{a}}$, Nuno Galamba ${ }^{\mathrm{a}, *}$ \\ ${ }^{a}$ Centre of Chemistry and Biochemistry and Biosystems and Integrative Sciences Institute, Faculty of Sciences of the \\ University of Lisbon, C8, Campo Grande, 1749-016 Lisbon, Portugal.
}

${ }^{*}$ Corresponding author. Electronic mail: njgalamba@fc.ul.pt 


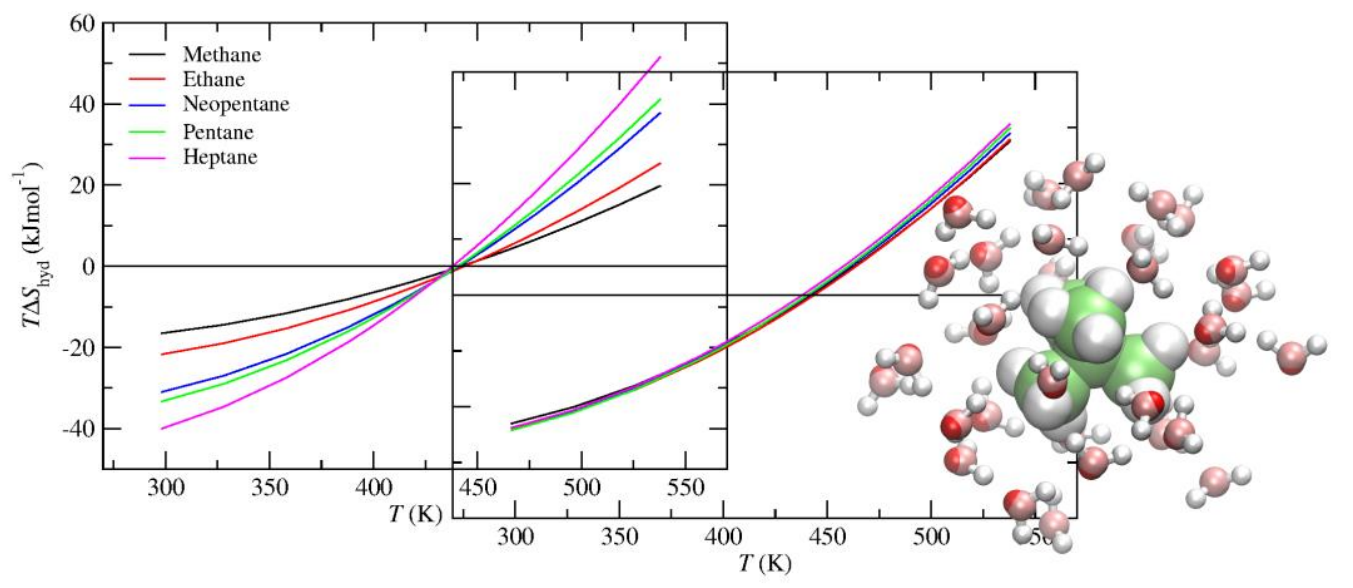

\section{Significance}

The hydrophobic effect, loosely defined as the disaffinity between oil and water, plays a pivotal role in many chemistry and biological phenomena, ranging from the low solubility of nonpolar molecules to protein folding. Here, we show, from molecular dynamics simulations, that the hydration entropy, normalized by the solvent accessible surface area, is nearly system size independent for hydrophobic, but not for amphiphilic molecules. A comprehensive molecular picture of the anomalous temperature dependence of hydrophobic hydration is then built upon information theory, through analysis of solute-water interactions and the reorganization of water's structure around hydrophobic groups. Kauzmann's "hydrocarbon model” on protein folding/denaturation is also discussed, with relatively large amphiphilic molecules exhibiting a more similar behavior to globular proteins, than hydrocarbons. 


\begin{abstract}
The hydrophobic effect plays a key role in many chemical and biological processes, including protein folding. Nonetheless, a comprehensive picture of the effect of temperature on hydrophobic hydration and protein denaturation remains elusive. Here, we study the effect of temperature on the hydration of model hydrophobic and amphiphilic solutes, through molecular dynamics, aiming at getting insight on the singular behavior of water, concerning the zero entropy temperature, $T_{S}$, and entropic convergence, also observed upon protein denaturation. We show that, similar to hydrocarbons and proteins, polar amphiphilic solutes exhibit a $T_{S}$, although strongly dependent upon solute-water interactions, opposite to hydrocarbons. Further, the temperature dependence of the hydration entropy, normalized by the solvent accessible surface area, is shown to be nearly solute size independent for hydrophobic, but not for amphiphilic solutes, for similar reasons. These results are further discussed in the light of information theory (IT) and the structure of water around hydrophobic groups. The latter shows that the tetrahedral enhancement of some water molecules around hydrophobic groups, associated with the reduction of water defects, leads to the strengthening of the weakest hydrogen bonds, relative to bulk water. However, a larger tetrahedrality is found in low density water populations, demonstrating that pure water has encoded structural information similar to that associated with hydrophobic hydration, consistent with IT assumptions. The source of the differences between Kauzmann's "hydrocarbon model" on protein denaturation and hydrophobic hydration is also discussed, with relatively large amphiphilic hydrocarbons displaying a more similar behavior to globular proteins, than aliphatic hydrocarbons.
\end{abstract}




\section{Introduction}

The hydrophobic effect, loosely defined as the disaffinity between oil and water, plays a central role in many chemical and biological processes, including protein folding and association(1-4). Hallmarks of hydrophobic hydration include a positive hydration free energy, $\Delta G_{\text {hyd }}$, or excess chemical potential $\left(\Delta \mu^{e x}\right)$, and an increase of the heat capacity, $\Delta C_{p}>0$, also observed upon protein denaturation $(5-8,4,3)$. The source of this $\Delta G_{\text {hyd }}>0$ is a negative hydration entropy, $\Delta S_{\text {hyd }}$, in spite of an also negative hydration enthalpy, $\Delta H_{\text {hyd }}$. Hydrophobic hydration is also characterized by an unusual temperature dependence, exhibiting (extrapolated) temperatures, $T_{S}$ and $T_{H}$, at which $\Delta S_{\text {hyd }}$ and $\Delta H_{\text {hyd }}$ are zero, respectively(4, 5, 7-10). Thus, above these temperatures, hydration would be favored, instead, by entropy and disfavored by enthalpy. A more puzzling aspect is the fact that the temperature at which $\Delta S_{\text {hyd }}$ is zero has been found to be similar, $\sim 400 \mathrm{~K}$, for different hydrocarbons(5, 7-10). $T_{s}$ was assessed for the transfer of hydrocarbons from the pure liquid to water, and shown to be similar to the temperature at which the hydrophobic contribution to the entropy of protein unfolding is zero(5, 7-10). The latter is apparently consistent with the analogy proposed by Kauzmann(11), between the exposure of the hydrophobic core of globular proteins to water, upon denaturation, and the transfer of a hydrocarbon from a nonpolar solvent into water. The transfer of a hydrophobic residue from water, to form the hydrophobic core of a protein, thus, interacting with other hydrophobic residues through van der Waals interactions, is then seen as the driving force (i.e., hydrophobic interactions) for protein folding(11-13). The resemblances between this "hydrocarbon model" and protein (un)folding have, however, long been questioned(4, 7, 1416).

Garde et al.(8) proposed an explanation for this convergence temperature, based on an information theory (IT), ascribing the system size independence of $T_{S}$ to the "weak temperature dependence of occupancy fluctuations for molecular scale volumes in water". They found a convergence temperature $\sim 410 \mathrm{~K}$, slightly below $T_{S}$ for model hard sphere solutes of sizes comparable to the noble gases and methane. A recent molecular simulation study by one of the authors, in turn, found a $T_{S}$ at $\sim 475 \mathrm{~K}$ for various model aromatic hydrocarbons(17) in liquid subcritical water at $100 \mathrm{~atm}$, coinciding with the convergence temperature.

The negative $\Delta S_{\text {hyd }}$ that characterizes hydrophobic hydration is believed to be associated with the work of cavity formation to insert the solute, whereas, the negative $\Delta H_{\text {hyd }}$ is related to solutewater interactions $(18,19)$, although alternative pictures have been proposed $(4,19-21)$. The solvent excluded volume, related to the formation of the cavity, induces a reorganization of water's 
hydrogen bond (HB) network. This cavity work was argued to be especially large because of the small size of water molecules, as opposed to organic solvents, with larger voids, thus, involving the reorganization of less molecules to create a suitable cavity $(19,22)$. Nonetheless, although more sharply defined in water, the most probable size cavities in hexane and dodecane were found to be about the same size as those for water(23). There should be an additional contribution to the entropy and enthalpy, related to the water reorganization around the cavity, due to solute-water interactions. Lee(19) proposed that the process of hydrophobic hydration could be broken into (a) the formation of a suitable cavity to lodge the solute and (b) the transfer of the solute into this cavity, involving the "turning on" of solute-water interactions. According to Lee(19), "changes in the thermodynamic quantities upon introduction of a cavity are entirely due to the solvent reorganization". In addition, the enthalpy and entropy contributions associated with the reorganization of water's HB network have been shown to nearly compensate, thus not influencing $\Delta G_{\text {hyd }} .(19,24-29)$ This water reorganization should, thus, be exclusively related to the solute insertion, and therefore, with solute-water interactions.

While not observed through neutron diffraction experiments(30-32) a tetrahedral enhancement of some water molecules next to small hydrophobic and amphiphilic molecules has been recently observed through molecular dynamics(17, 33-41), Raman scattering measurements with multivariate curve resolution $(42,43)$ and infrared spectroscopy $(44,45)$. Notice that any structural enhancement related to the reorganization of water upon insertion of the solute, while not contributing to the free energy (enthalpy-entropy compensation), still contributes to the hydration entropy and enthalpy. Nonetheless, disentangling the possible contributions from the cavity alone and from the solute-water interactions, to this tetrahedral enhancement, is not straightforward. Water molecules without interstitial water neighbors ("water defects" associated with the existence of a fifth water neighbor in the interstitial region in-between the first and second coordination spheres) are more tetrahedral than water molecules with defects(46). Thus, since larger cavities should be found near water molecules with a lower number of neighbors (i.e., lower density), similar to water next to nonpolar solutes, where the fifth water neighbor is already well beyond the girth of the first coordination sphere(17), this suggests that this tetrahedral enhancement should already arise with the formation of the cavity, thus contributing to both, $\Delta S_{\text {hyd }}$ and $\Delta G_{\text {hyd }}$. This putative contribution is, however, generally neglected, and scale particle theory(47, 19, 3) (SPT), Pratt and Chandler's theory $(48)$, and IT $(8,49)$, quantitatively account for the $\Delta G_{\text {hyd }}$ of small hard spheres, without regarding any structural enhancement of water around the solute(50). Thus, IT, for instance, can quantitatively describe $\Delta G_{\text {hyd }}$ for rare gases, based exclusively on the oxygen-oxygen radial distribution function and the density of neat water(49). We anticipate that a larger tetrahedral 
enhancement in pure water populations, without interstitial water molecules, was observed in this study, relative to water next to hydrophobic solutes. Thus, any structural enhancement, related with the solute, is already found in pure water due to structural fluctuations (or heterogeneities).

Following the above discussion, concerning the cavity work, $\Delta S_{\text {hyd }}$ should decrease (more negative) with the solute size. This result is consistent with molecular simulations and is reproduced by various theories, including SPT and IT. Entropic convergence at $T_{S}$ implies, however, the reversal of this size dependence, with larger solutes exhibiting larger, positive, $\Delta S_{\text {hyd }}$, at $T \geq T_{S}$. This, in turn, suggests that the temperature dependence of $\Delta S_{\text {hyd }}$ cannot be explained by the cavity work alone, even if suitable cavities for an arbitrarily large solute, formed in water around this temperature. Thus, although this crossover is predicted by IT for hard spheres, a molecular-level understanding of this positive entropy and its system size dependence remains elusive. The fact that the hydration entropy of polar and non-polar solutes of similar size (e.g., methane and methanol)(51) exhibit a different temperature dependence, suggests that solute-water and/or waterwater interactions are important to explain $\Delta S_{\text {hyd }}(T)$, and, therefore, the molecular origin of $T_{S}$ in hydrophobic solutes and proteins. For instance, while $\Delta S_{\text {hyd }}$ of methane and methanol are similar near the melting point of water, the entropy of the former increases at a higher rate with the temperature(51).

Here, we study the hydration thermodynamics of model hydrocarbons and amphiphilic molecules, as simple prototypes of the core of globular proteins, aiming at understanding the molecular source of $T_{S}$ of hydrophobic solutes and proteins in water. Further, solvation analysis is carried out to probe the effects of temperature and solute size on the HB network of water, next to hydrophobic and amphiphilic solutes.

\section{Methods}

Molecular dynamics (MD) simulations of various OPLS-aa(52) model hydrocarbons and alcohols in water were performed with the program GROMACS 5.1.4(53). The following solutes were studied: methane, ethane, neopentane, pentane, heptane, benzene, naphthalene, anthracene, pyrene, methanol, ethanol, and neopentyl alcohol (neopentanol), chosen to include aliphatic and aromatic hydrocarbons as well as amphiphilic solutes of similar sizes. The hydration free energies of some solutes were first calculated at $298 \mathrm{~K}$ and 1 atm for distinct water models, namely, TIP3P(54), SPC/E(55), TIP4P/Ew(56), and TIP4P/2005(57), for comparison purposes. Although the TIP3P water model provides in general more accurate $\Delta G_{\text {hyd }}$ values, the TIP4P/2005 water gives the most accurate free energies among the other water models (see Table S1). Thus, because it 
provides a better description of liquid water, than TIP3P, the simulations were carried out with the TIP4P/2005 model.

To probe the hydration free energy maxima, the simulations were carried out at 100 atm allowing observing this maximum below the boiling temperature of water at this pressure ( $\sim 580 \mathrm{~K})$ for most systems. The aqueous systems were comprised of a single molecule of solute and 1000 water molecules in a cubic box with periodic boundary conditions. The systems were first equilibrated in the $(N, P, T)$ ensemble for $10 \mathrm{~ns}$ after an $100 \mathrm{ps}$ simulation in the $(N, V, T)$ ensemble. The $T$ and $p$ were controlled with the thermostat of Bussi et al.(58) and the Parrinello-Rahman barostat(59), and the equations of motion were solved with the Verlet leap-frog algorithm with a 2 fs time-step. Electrostatic interactions were computed via the particle-mesh Ewald (PME) method(60). A cut-off of $1 \mathrm{~nm}$ was used for non-bonded van der Waals and for the PME real space electrostatic interactions. Heavy atom-hydrogen covalent bonds were constrained with the LINCS algorithm(61).

The hydration free energies were obtained through "alchemical" free energy calculations(62) with the Bennett acceptance ratio (BAR)(63) method. The method involves the perturbation of the system based on the definition of a parameter, $\lambda$, taking values in the interval $[0,1]$, allowing connecting the end states of interest, $\mathrm{A}(\lambda=1)$ and $\mathrm{B}(\lambda=0)$, defined by the Hamiltonians $\mathbf{H}_{A}(r, p ; \lambda)$ and $\mathbf{H}_{B}(r, p ; \lambda)$. The transition from state $A$, the solution, to state $\mathrm{B}$, the solvent, is performed by a number $\left(N_{\lambda}\right)$ of different values of $\lambda$, corresponding to non-physical states. The $\Delta G_{\text {hyd }}$ calculated in this work, concern the transfer of a solute from a fixed position in the gas phase to a fixed position in water, following Ben-Naim and Marcus(64) standard, as opposed to the transfer from a non-polar environment to water.

A decoupling approach was used with $N_{\lambda}=20$, connecting the states $A$ and $B$. For the Coulombic interactions decoupling, a $\Delta \lambda=0.25$ was adopted, whereas for the van der Waals interactions a $\Delta \lambda=0.05$ was used. Langevin stochastic $\mathrm{MD}(65)$ was carried out and a soft-core potential was used for Lennard-Jones and electrostatic interactions to avoid numerical singularities at terminal $\lambda$ values, with $\alpha=0.5, \sigma=0.3$, and a soft-core power of 1.(62, 66-69) The simulations for each $\lambda$ consisted of a steepest descent energy minimization step, followed by a $0.5 \mathrm{~ns}$ Langevin NVT simulation, and a $1 \mathrm{~ns}$ Langevin simulation in the NPT ensemble, using the Parrinello-Rahman barostat(70). The hydration free energy was then computed from 2 independent Langevin NPT simulations, $10 \mathrm{~ns}$ long, for each $\lambda$. For some temperatures, where larger differences were observed, typically near the $\Delta G_{\text {hyd }}$ maxima, up to 5 independent simulations, $10 \mathrm{~ns}$ long, were carried out.

The entropy was assessed from, $\Delta S_{\text {hyd }}=-\left(\partial \Delta G_{\text {hyd }} / \partial T\right)_{p}$, where $\Delta G_{\text {hyd }}$ was fitted to a second 
order polynomial, and the hydration enthalpy was estimated from, $\Delta H_{\text {hyd }}=\Delta G_{\text {hyd }}+T \Delta S_{\text {hyd }}$. Every approach to assess either $\Delta H_{\text {hyd }}$ or $\Delta S_{\text {hyd }}$, suffers from limitations $(62,71)$ and, therefore, a larger uncertainty is associated with the hydration entropy and enthalpy.

Solvent accessible surface areas (SASA) were computed by rolling a solvent sphere $(72,73)$ of radius $1.4 \AA$ over the van der waals surface(74) of the solutes.

The tetrahedrality of water was assessed through the calculation of the orientational order parameter(75), $q$, in the rescaled form(76), $q=1-\frac{3}{8} \sum_{i=1}^{3} \sum_{j=i+1}^{4}\left(\cos \theta_{i j}+1 / 3\right)^{2}$, where $\theta_{i j}$ is the angle formed by the lines joining the $\mathrm{O}$ atom of a given water molecule and those of its nearest neighbors, $i$ and $j$. The average value of $q$ varies between 0 (ideal gas) and 1 (perfect tetrahedral HB network).

\section{Results}

\section{Thermodynamic Parameters}

The temperature dependence of $\Delta G_{\text {hyd }}, \Delta S_{\text {hyd }}$, and $\Delta H_{\text {hyd }}$ for the aliphatic hydrocarbons is shown in Fig. 1. The temperature of the $\Delta G_{\text {hyd }}$ maximum, $\sim 440 \mathrm{~K}$, is nearly solute size independent, consistent with experimental data extrapolations at $1 \mathrm{~atm}$, although for the transfer of a hydrocarbon from the pure liquid, rather than from the gas phase, into water(5, 7). A minor effect of the pressure on $\Delta G_{\text {hyd }}$ of methane is observed, indicating that our results should be comparable to those extrapolated at 1 atm. $T_{S}$ is the temperature at which $\Delta G_{\text {hyd }}$ displays a maximum and, thus, $\Delta S_{\text {hyd }}=0$. For $\Delta G_{\text {hyd }}$ of the form of a second order polynomial (see Fig. 1(a 1 )),

$$
\Delta G_{\mathrm{hyd}}=a+b T+c T^{2}
$$

this temperature is given by $T_{S}=-b / 2 c$. The $T_{S}$ obtained through this equation for methane, ethane, neopentane, pentane, and heptane are, respectively, 442K, 444K, 441K, 441K, and $439 \mathrm{~K}$. Thus, entropic convergence is observed at $\sim 440 \pm 10 \mathrm{~K}$, because of the $T_{S}$ near solute size independence. The temperature dependence of $\Delta S_{\text {hyd }}$ and $\Delta H_{\text {hyd }}$ for methane at 1 atm was also assessed, allowing comparing with available "experimental" (see Fig. S1) data(51, 77, 78). A good agreement is observed. Furthermore, a $T_{S}$ of $412 \mathrm{~K}$ (extrapolated), close to the experimental, $400 \mathrm{~K}$, was found, at $1 \mathrm{~atm}$. 

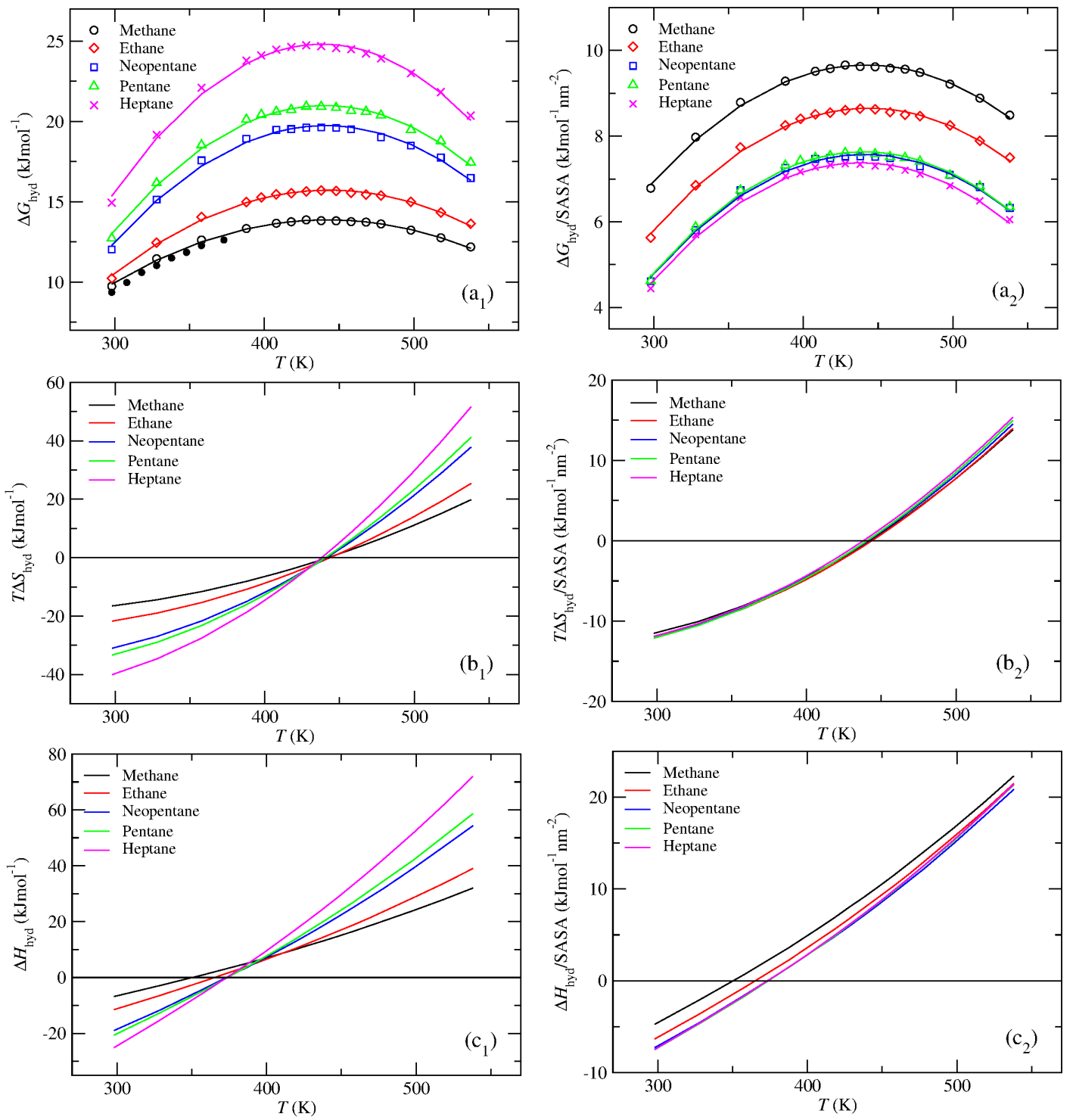

Figure 1 - Temperature dependence of $\left(\mathrm{a}_{1}\right) \Delta G_{\mathrm{hyd}},\left(\mathrm{b}_{1}\right) \Delta S_{\mathrm{hyd}}$, and $\left(\mathrm{c}_{1}\right) \Delta H_{\mathrm{hyd}}$, for the distinct hydrocarbons, at $100 \mathrm{~atm}$. The $\Delta S_{\text {hyd }}$ convergence region extends around $\sim 440 \pm 10 \mathrm{~K}$. The filled circles in $\left(\mathrm{a}_{1}\right)$ are for methane at $1 \mathrm{~atm}$. The entropy was obtained from the temperature derivative of the free energy, fitted to a second order polynomial, displayed as solid curves in $\left(\mathrm{a}_{1}\right)$. The standard deviations of the free energy obtained from the independent simulations are comparable to the symbols' size and are omitted. The same plots, normalized by the SASA (see Table S2) are shown, respectively, in $\left(\mathrm{a}_{2}\right),\left(\mathrm{b}_{2}\right)$, and $\left(\mathrm{c}_{2}\right)$.

Fig. 1(b $\left.\mathrm{b}_{1}\right)$ shows that the hydration entropy (i.e., $\left.T \Delta S_{\mathrm{hyd}}\right)$ at $298 \mathrm{~K}$ decreases (more negative) with the solute size, consistent with the fact that a larger cavity must form to host larger solutes. However, the rate of increase of the entropy with the temperature increases with the solute size, and above $\sim T_{S}$ an inversion occurs, with $\Delta S_{\text {hyd }}$ increasing (more positive) with the solute size. Thus, the fact that $\Delta S_{\text {hyd }}>0$ and that $\Delta S_{\text {hyd }}$ increases with the solute size above $T_{S}$, suggests that $\Delta S_{\text {hyd }}$ should no longer be governed by the cavity work.

$\Delta H_{\text {hyd }}$ exhibits a similar behavior, although different $T_{H}$ are found, the latter increasing with the solute size. Furthermore, $T_{H}$ is lower than $T_{S}$ for every solute. The solute size dependence of the rate 
of increase of the hydration enthalpy with the temperature (i.e., $\left.\Delta C_{p}=\left(d \Delta H_{\text {hyd }} / d T\right)_{p}\right)$ is consistent with the temperature dependence of the solute-water van de Waals interactions (see Fig. S2). However, unlike $\Delta H_{\text {hyd }}$, solute-water van der Waals interactions do not converge at $\sim 400 \mathrm{~K}$, suggesting that the reversal of the solute size dependence of $\Delta H_{\text {hyd }}$ should be further connected with water-water interactions. Electrostatic interactions are significantly weaker and appear to exhibit a convergence behavior with the temperature; interestingly, for methane and ethane, the solute-water Coulombic repulsion first decreases with the temperature, opposite to the larger solutes (see Fig. S2).

Figure 1 also shows the same thermodynamic parameters normalized by the solvent accessible surface areas (SASA). Remarkably, the $T \Delta S_{\text {hyd }} / S A S A$ exhibits a nearly solute size independent behavior, while the rate of increase of $\Delta H_{\text {hyd }} / S A S A$ is now more similar for the distinct solutes (see also Fig. S2(b)). Notice that, in addition to a curvature homogenization of $\Delta G_{\text {hyd }} / S A S A$, a reversal of the system size dependence occurs (see Figs 1(a1) and 1(a2)). Figure 1(b2) shows that the SASA allows normalizing the solute size dependence of the entropy in both regimes, $\Delta S_{\text {hyd }}\left(T<T_{S}\right)<0$ and $\Delta S_{\text {hyd }}\left(T>T_{S}\right)>0$. However, because the SASA encloses information on the volume of the cavity and on the extent of solute-water interactions and HB perturbations, it does not allow distinguishing between the temperature dependence contributions associated with the cavity work and solute-water interactions.

Notice that $\Delta G_{\text {hyd }}$ grows linearly with the solute volume, for small solutes, whereas for large hydrophobic surfaces (not studied in this work) it grows linearly with the surface area(2). This behavior is believed to be associated with the formation of a liquid-vapor like interface next to large (> $\sim 1 \mathrm{~nm}$ ) solutes, with water molecules moving away from the solute (i.e., microscopic dewetting)(2), opposite to small solutes. The structural transformations of water and their putative connection with the distinct rates of increase of $\Delta S_{\text {hyd }}$ and $\Delta H_{\text {hyd }}$, with the temperature, are discussed in sub-section 2. Before, however, we discuss the results for the model aromatic hydrocarbon and alcohol aqueous systems.

Figure 2 shows similar plots for the aromatic hydrocarbons, exhibiting a $T_{S} \sim 470 \mathrm{~K}$, thus, $\sim 30$ $\mathrm{K}$ larger than for the aliphatic hydrocarbons. This temperature is similar to that recently observed by one of the authors(17) for the generalized amber force field(79) model of benzene, naphthalene, and anthracene, in TIP4P-Ew(56) water. The $T_{S}$ obtained from the second order polynomial fit for benzene, naphthalene, anthracene, and pyrene, are respectively, $469 \mathrm{~K}, 472 \mathrm{~K}, 474 \mathrm{~K}$, and $476 \mathrm{~K}$. A similar qualitative behavior to that displayed in Fig. 1 is observed for $\Delta S_{\text {hyd }}$ and $\Delta H_{\text {hyd }}$, normalized 
by the SASA. Generalized curves for $\triangle S_{\text {hyd }} / S A S A$ of aliphatic and aromatic hydrocarbons are given in Fig. S3 and Table S3. The higher rate of increase of $\Delta H_{\text {hyd }}$ with the solute size is again consistent with the respective rate of increase of the solute-water van der Waals interactions (see Fig. S4). However, electrostatic interactions are now more important, significantly contributing to $\Delta H_{\text {hyd }}$ and, therefore, to $\Delta G_{\text {hyd }}$. Thus, opposite to aliphatic hydrocarbons a reversal of the system size dependence is not observed for $\Delta G_{\text {hyd }} / S A S A$, relative to $\Delta G_{\text {hyd }}$. This is because $\Delta G_{\text {hyd }}$ is now governed by $\Delta H_{\text {hyd }}$, via solute-water interactions. Notice that the solute size dependence of $\Delta G_{\mathrm{hyd}} / S A S A$, unlike $\Delta G_{\mathrm{hyd}}$, is governed by $\Delta H_{\mathrm{hyd}} / S A S A$, for both aliphatic and aromatic hydrocarbons, in the sense that $\Delta S_{\text {hyd }} / S A S A$ is nearly solute size independent. For pyrene $\left(\mathrm{C}_{16} \mathrm{H}_{10}\right)$, electrostatic interactions are less repulsive than for anthracene $\left(\mathrm{C}_{14} \mathrm{H}_{10}\right)$, because of the higher rate of carbon/hydrogen atoms, explaining the similar rate of increase of $\Delta H_{\text {hyd }}$ and, thus, the nonconvergence of the enthalpy at high temperatures (see Fig. 2(c1)). 

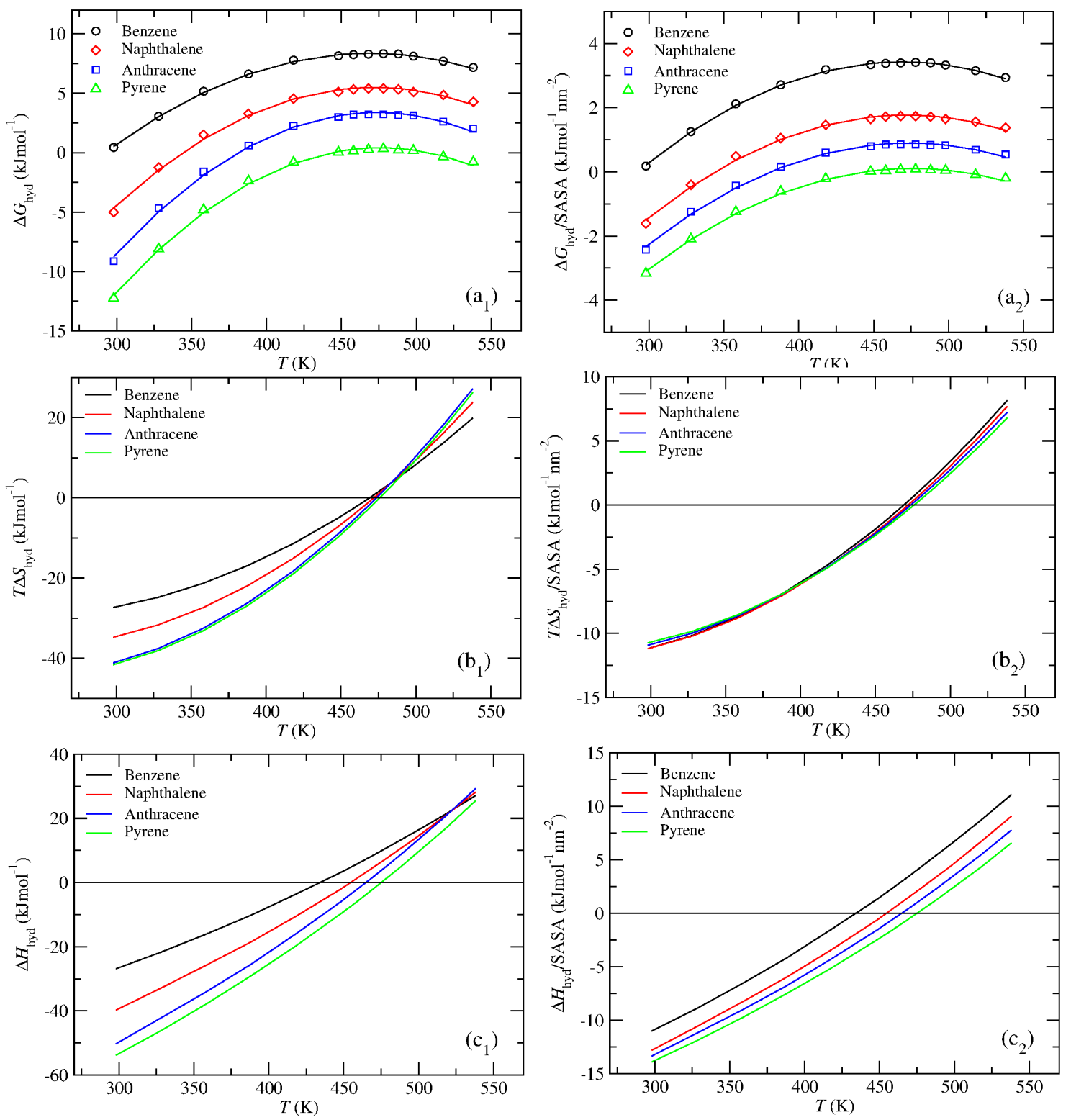

Figure 2 - Temperature dependence of $\left(\mathrm{a}_{1}\right) \Delta G_{\text {hyd }}$, ( $\left.\mathrm{b}_{1}\right) \Delta S_{\text {hyd }}$, and $\left(\mathrm{c}_{1}\right) \Delta H_{\text {hyd }}$, of benzene, naphthalene, anthracene, and pyrene at $100 \mathrm{~atm}$. The entropy was obtained from the temperature derivative of the free energy fitted to a second order polynomial displayed as solid curves in $\left(a_{1}\right)$. The same plots, normalized by the SASA (see Table S2) are shown, respectively, in $\left(a_{2}\right),\left(b_{2}\right)$, and $\left(c_{2}\right)$.

The $\Delta S_{\text {hyd }}$ increase with the temperature for both aliphatic and aromatic solutes indicates a facilitated insertion of the solute, which could be explained by the larger number of broken HBs in water, increasing the size of the cavities, and thus, the probability of finding a suitable cavity to lodge the solute. For hard spheres this probability is related to the hydration free energy, by $\Delta G_{\text {hyd }}=-k_{B} T \ln p_{0}(R)$, where $R$ is the solvent accessible radius, given by $R=R_{S}+R_{W}$, and $R_{S}$ and $R_{W}$ are the radius of the solute and water, modeled as hard spheres, whereas $p_{0}(R)$ is the probability that a sphere of radius $R$ randomly inserted in water is devoid of water molecules $(3,8,80)$.

Garde et al.(8) analyzed the entropy convergence based on an information theory where the hydration free energy of small hard spheres is approximated by a function of $T \rho^{2}(T)$, 


$$
\begin{aligned}
& \Delta G_{\text {hyd }}^{\mathrm{HS}}=T \rho^{2}\left\{k_{B} v^{2} / 2 \sigma^{2}\right\}+T\left\{k_{B} \ln \left(2 \pi \sigma^{2}\right) / 2\right\} \\
& \Delta G_{\text {hyd }}^{\mathrm{HS}}=T \rho^{2}(T) x(v)+T y(v)
\end{aligned}
$$

Equation (2) was obtained from the probability of finding exactly $n$ solvent molecules in the cavity volume $v=4 \pi R^{3} / 3$, modeled by a Gaussian distribution, $p_{n} \approx \exp \left(-\delta n^{2} / 2 \sigma^{2}\right) / \sigma \sqrt{2 \pi}$, with variance, $\sigma^{2}=\left\langle\delta n^{2}\right\rangle$ and $\delta n=n-\langle n\rangle$. From the second equation, $x(v)$ and $y(v)$ only depend on the excluded volume.(8) This equation follows from the observation by Garde et al.(8) of a weak temperature dependence of occupancy fluctuations for solute excluded volumes in water, $\left(\sigma^{2}(T, v) \approx \sigma^{2}(v)\right)$ not expected in non-polar organic solvents. The latter offers an explanation to the singular ability of water to exhibit a zero entropy convergence temperature, contradicting the view that significantly larger voids should form in water associated with a more broken HB network. Notice the above equation does not depend on any specific structural transformation of water around the solute, neither associated with volume exclusion nor with solute-water interactions, but rather only on the density and density fluctuations of water with the temperature. The hydration or excess entropy obtained from eq. (2) gives,

$$
\Delta S_{\text {hyd }}=\rho^{2} x(v)(2 T \alpha-1)-y(v)
$$

where $\alpha$ is the thermal expansion coefficient. Now, the temperature of zero entropy is given by,

$$
T_{S}^{H S}=\frac{y(v)+\rho^{2} x(v)}{2 \rho^{2} x(v) \alpha}=\frac{b^{H S}}{2 c^{H S}}
$$

which has a similar form to the empirical $T_{S}$ obtained from eq. (1), i.e., $T_{S}=-b / 2 c$.

The empirical parameters $b$ and $c$ in eq. (1) contain information on the density fluctuations of water as well as on the solute-water interactions, absent in $b^{H S}$ and $c^{H S}$. The inclusion of van der Waals interactions is expected to shift $T_{S}^{H S}$ to higher temperatures(3). However, the normalization of $\Delta S_{\text {hyd }}$, defined through eq. (3), by the SASA, should still exhibit a solute size dependence, nearly absent in hydrocarbons. This suggests that $\Delta S_{\text {hyd }}(T)$ should depend not only on the size of the cavity and density fluctuations, accounted by IT, but also on solute-water interactions, both related with the SASA. In addition, whereas $T_{S}$ is solute size independent, it should be determined by water's density fluctuations and solute-water interactions. This point is further discussed below.

Turning attention to the model amphiphilic solutes, Fig. 3 shows the thermodynamic functions for methanol, ethanol, phenol, and neopentanol. The temperature dependence of $\Delta S_{\text {hyd }}$ and $\Delta H_{\text {hyd }}$ for methanol, at 1 atm, was also assessed, allowing comparing with available "experimental" (see Fig. S1) data(51, 81, 82); a reasonable agreement is found, in spite of significant differences in the curvature of $\Delta S_{\text {hyd }}$. 

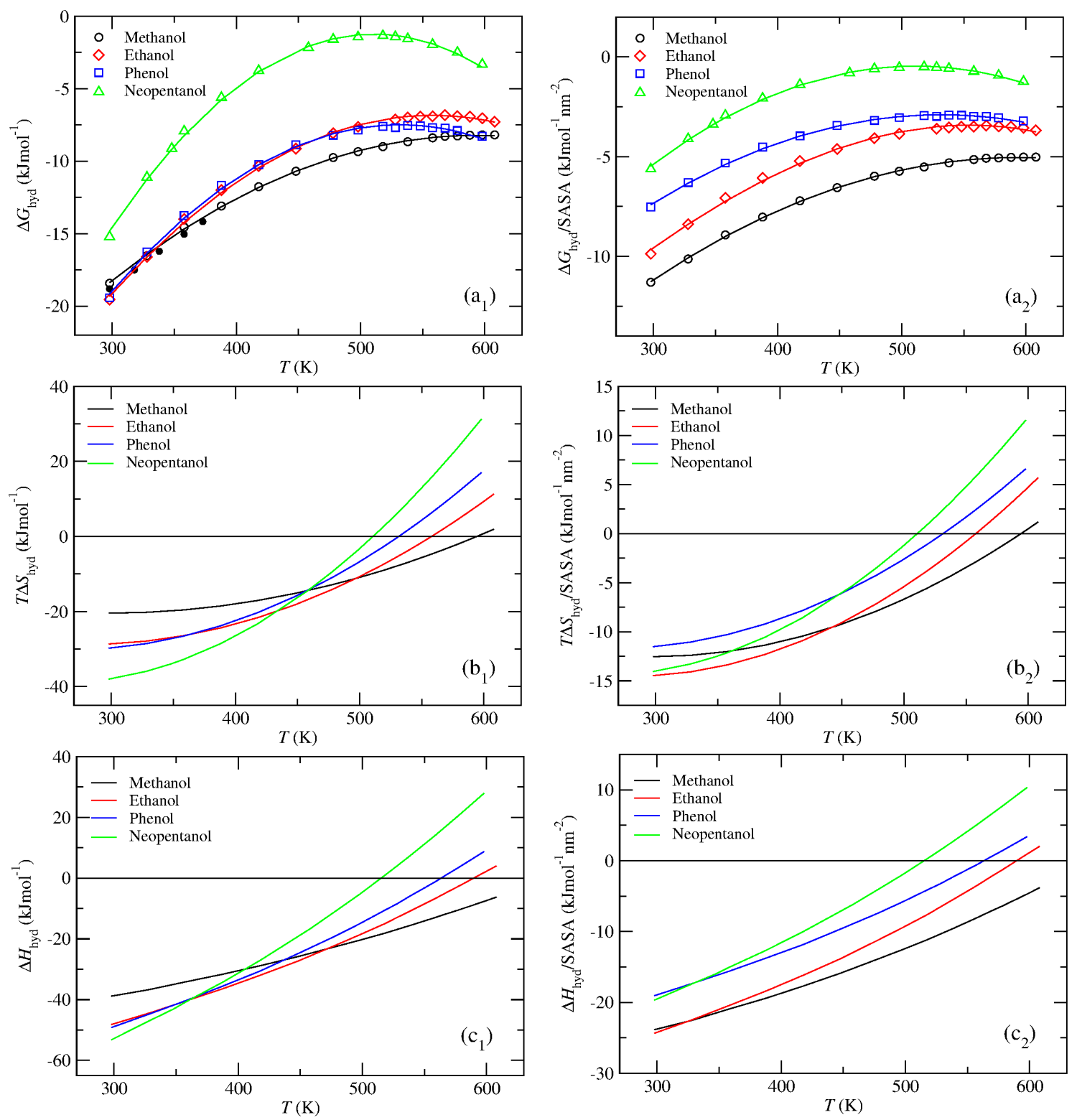

Figure 3 - Temperature dependence of $\left(\mathrm{a}_{1}\right) \Delta G_{\text {hyd }},\left(\mathrm{b}_{1}\right) \Delta S_{\text {hyd }}$, and $\left(\mathrm{c}_{1}\right) \Delta H_{\text {hyd }}$ of methanol, ethanol, phenol, and neopentanol at $100 \mathrm{~atm}$. The filled circles in (a) are for methanol at $1 \mathrm{~atm}$. The entropy was obtained from the temperature derivative of the free energy fitted to a second order polynomial displayed as solid curves in $\left(a_{1}\right)$. The same plots, normalized by the SASA (see Table S2) are shown, respectively, in $\left(a_{2}\right),\left(b_{2}\right)$, and $\left(\mathrm{c}_{2}\right)$.

The solutes exhibit distinct $T_{S}$, opposite to hydrocarbons. Furthermore, $T_{S}$ are significantly larger than for the hydrocarbons. The $T_{S}$ obtained from the second order polynomial fit for methanol, ethanol, phenol, and neopentanol, are respectively, $594 \mathrm{~K}, 557 \mathrm{~K}, 531 \mathrm{~K}$, and $511 \mathrm{~K}$.

Entropic convergence can be observed at $\sim 458 \mathrm{~K}$, with the exception of ethanol. Electrostatic interactions are now dominant (see Fig. S5) and these are stronger for methanol and ethanol than for phenol and neopentanol, opposite to van der Waals interactions.

In spite of the apparent solute size dependence (see Fig. 3(b1)), this is not the reason for the different $T_{S}$, since a near system size independence was already demonstrated for hydrocarbons. To 
further demonstrate this point we calculated $\Delta G_{\text {hyd }}, \Delta S_{\text {hyd }}$, and $\Delta H_{\text {hyd }}$ for neopentane, benzene, and neopentanol, neglecting electrostatic interactions. Thus, non-bonded solute-water interactions were restricted to van der Waals interactions. Figure 4 shows that a similar $T_{S}$ is now obtained for the distinct solutes. A $T_{S}$ of $442 \mathrm{~K}, 449 \mathrm{~K}$ and $442 \mathrm{~K}$ were found, respectively, for neopentane, benzene, and neopentanol modeled exclusively by van der Waals interactions. This indicates that the different $T_{S}$ are connected with the solute-water interactions, especially, electrostatic interactions, almost absent in aliphatic hydrocarbons.

Furthermore, the solute size dependence of $\Delta S_{\text {hyd }} / S A S A$, not observed for hydrocarbons, is connected with the fact that electrostatic interactions are less sensible to the SASA, since these are long-ranged.
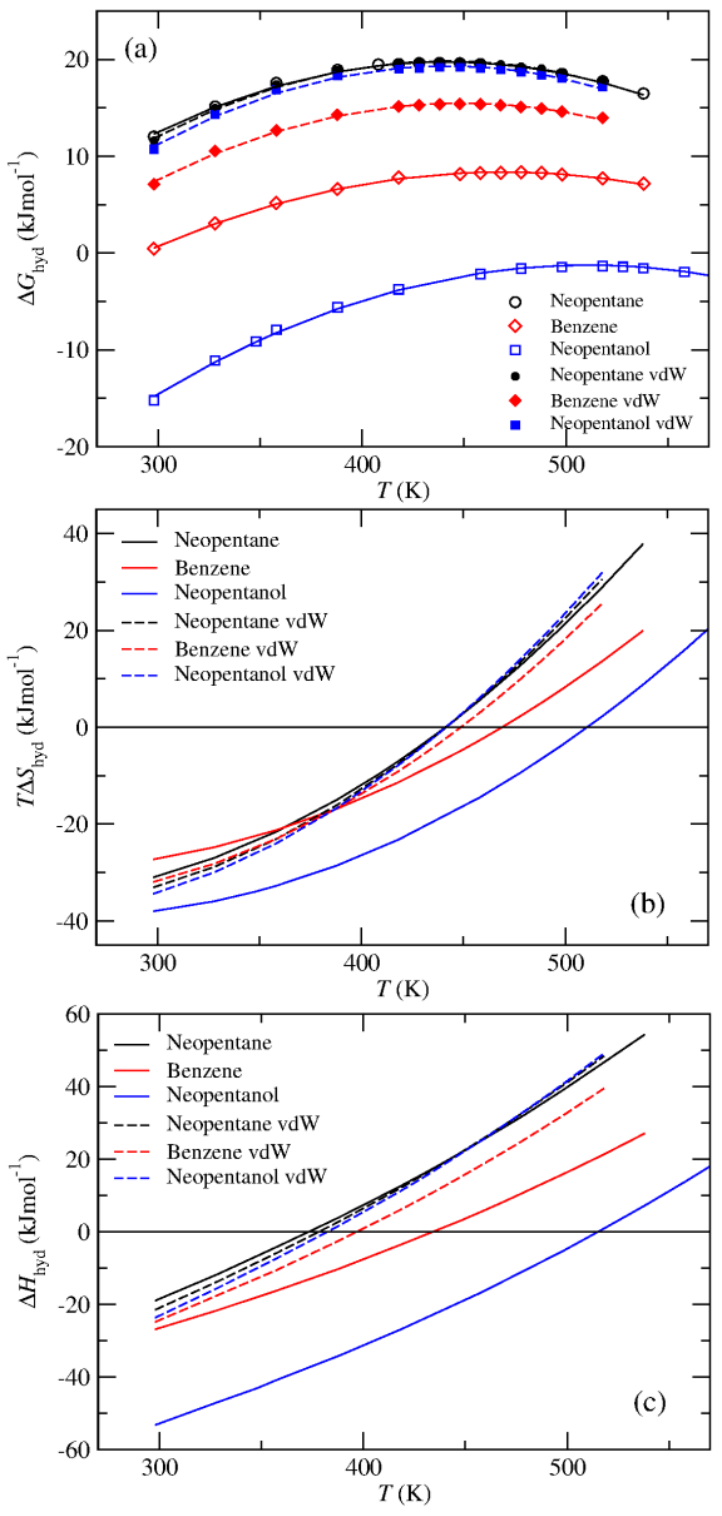

Figure 4 - Temperature dependence of the (a) $\Delta G_{\text {hyd }}$, (b) $\Delta S_{\text {hyd }}$, and (c) $\Delta H_{\text {hyd }}$ of model neopentane, benzene, and neopentanol at $100 \mathrm{~atm}$, with and without (vdW) including solute-water electrostatic interactions. 
Now, opposite to the aliphatic hydrocarbons it can be seen that $T_{H}$ is larger than $T_{S}$ for the amphiphilic solutes, with the exception of neopentanol. A distinction between protein denaturation and the "hydrocarbon model" is the fact that a smaller difference is observed between $T_{S}$ and $T_{H}$ for proteins, relative to hydrocarbons $(4,10,83)$. The latter reflects, therefore, differences between a hydrophobic group in the core of a globular protein and a hydrocarbon dissolved in a nonpolar solvent. In spite of considering the transfer from the gas phase to water, distinct regimes can be observed in Figs 1-3, namely, $T_{S}>T_{H}$ for aliphatic hydrocarbons, $T_{S} \sim T_{H}$ for the larger polycyclic aromatic hydrocarbons and neopentanol, and $T_{S}<T_{H}$ for the smaller alcohols. This suggests that the interior of a protein lies in-between a hydrophobic and a small amphiphilic system, consistent with the fact that the core of globular proteins is not exclusively populated by aliphatic residues.

\section{Solvation Analysis}

In the previous section we analyzed the differences between the temperature dependence of hydrophobic and amphiphilic hydration with respect to solute-water interactions and solute size dependence. These solute-water interactions, and water-water interactions, neglected until now, depend on the structural transformations of water near the solutes. Thus, we now discuss such structural transformations.

In the following we separate solvation water molecules, that is, those in the first hydration shell of the solutes, defined by the first minimum of the radial distribution functions, in water molecules with 4 or more water neighbors $(4 \mathrm{MWN})$ and water molecules with less than 4 water neighbors (L4WN). The first population remains with a nearly tetrahedral coordination, comparable to bulk water, whereas the second can form up to a maximum of $3 \mathrm{HBs}$, resembling water molecules near a water-vapor interface(84-87).

Figure 5(a) shows a tetrahedrality enhancement of water molecules with 4MWN, next to neopentane, relative to bulk water; similar results were observed for the other solutes. Interestingly, an even larger tetrahedral enhancement is observed for water molecules with no interstitial water molecules (NIWM) (see Fig. 5(b)); an interstitial water molecule was defined as a fifth water neighbor found up to $3.7 \AA(88)$, around a central water molecule. This demonstrates that the structural transformations of water associated with the hydration of small hydrophobic solutes already occur spontaneously in pure water, due to density fluctuations. Thus, any (compensating) entropic and enthalpic contributions associated with this water reorganization are already encoded in pure water, consistent with the fact that IT only requires information on the pair distribution function and density fluctuations of pure water.

The hydrophobic related tetrahedral enhancement (Fig. 5(a)), consistent with previous 
simulation(33, 34, 36, 17, 37, 38, 40, 41) and experimental(42-44) studies for different hydrophobic molecules and groups, but at odds with neutron diffraction experiments(30-32), is completely lost at high temperatures for methane and neopentane, but not for methanol and neopentanol (see Fig. 5b).
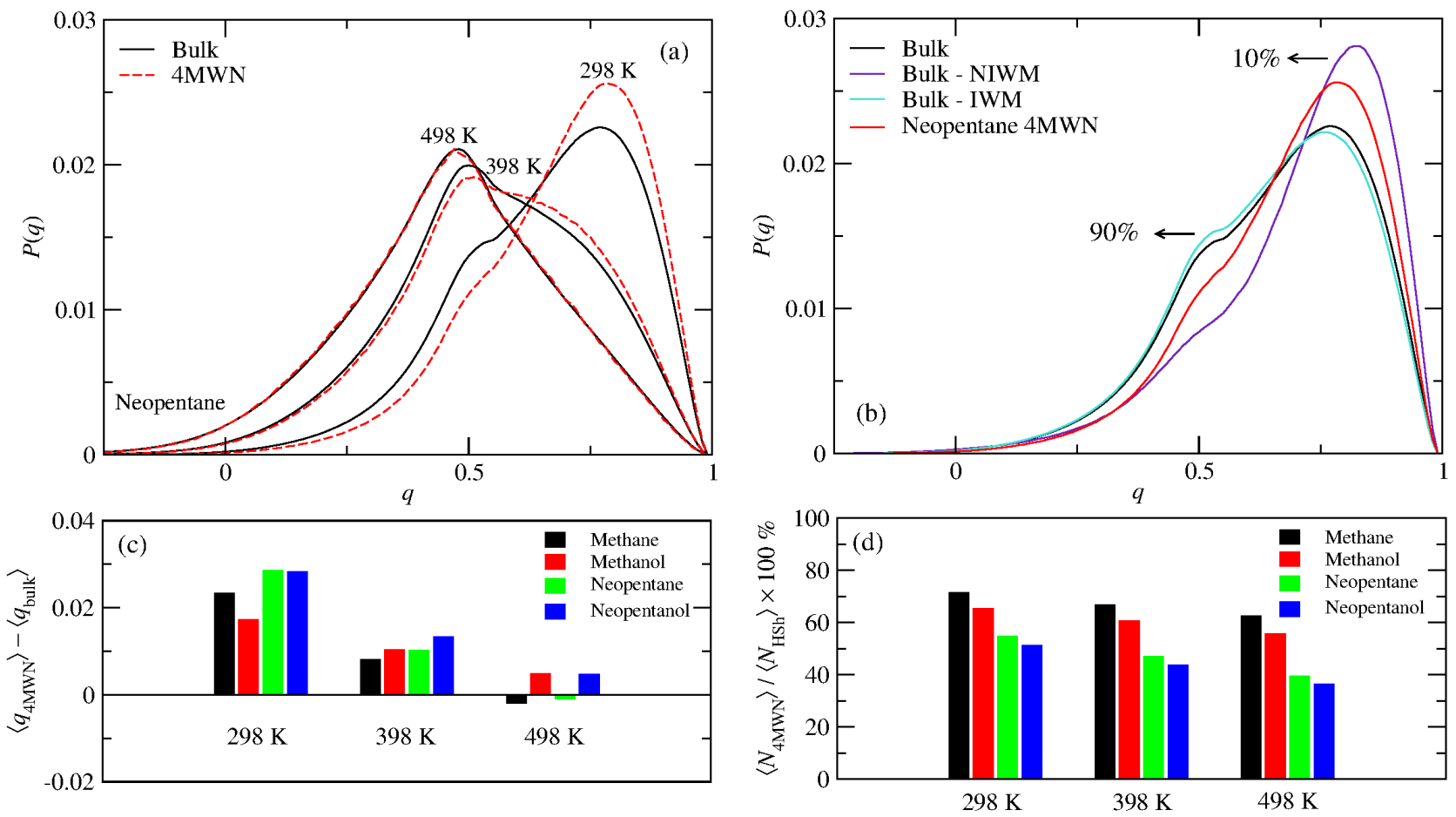

Figure 5 - Temperature dependence of the (a) tetrahedrality $(q)$ distributions of water molecules with 4 or more water neighbors (4MWN) in the first coordination sphere of the methyl groups of neopentane and in bulk water, (b) comparison between $q$ distributions in the neopentane coordination sphere and water molecules with no interstitial water molecules (NIWM) and with interstitial water molecules (IWM) in pure water at $298 \mathrm{~K}$; the respective populations are shown (c) tetrahedrality difference between the water population with $4 \mathrm{MWN}$ and bulk water, and (d) fraction of the water population in the coordination sphere with 4MWN.

A similar structural loss is observed in the NIWM population at high temperatures, exhibiting a lower tetrahedrality than the IWM population (see Table S4). This is connected with the fact that at high temperatures water loses much of its tetrahedral geometry and a fifth water molecule increases the number of ways in which a tetrahedron can be formed(46). Figure 5(d) shows the temperature dependence of the fraction of water molecules with 4MWN. This is larger for the smaller solutes, consistent with the fact that the HB network of water is less perturbed upon dissolution of a small solute.

The observed tetrahedral enhancement leads to the formation of stronger HBs, especially with the third and fourth nearest water neighbors, as shown in Fig. 6; similar results were recently found by one of the authors for aromatic solutes(17). Notice that the L4WN population exhibits the stronger $\mathrm{HB}$, with the nearest neighbor. However, pair interactions with the third nearest neighbor 
are already weaker than in the in the bulk, even at room $T$, among this water population.
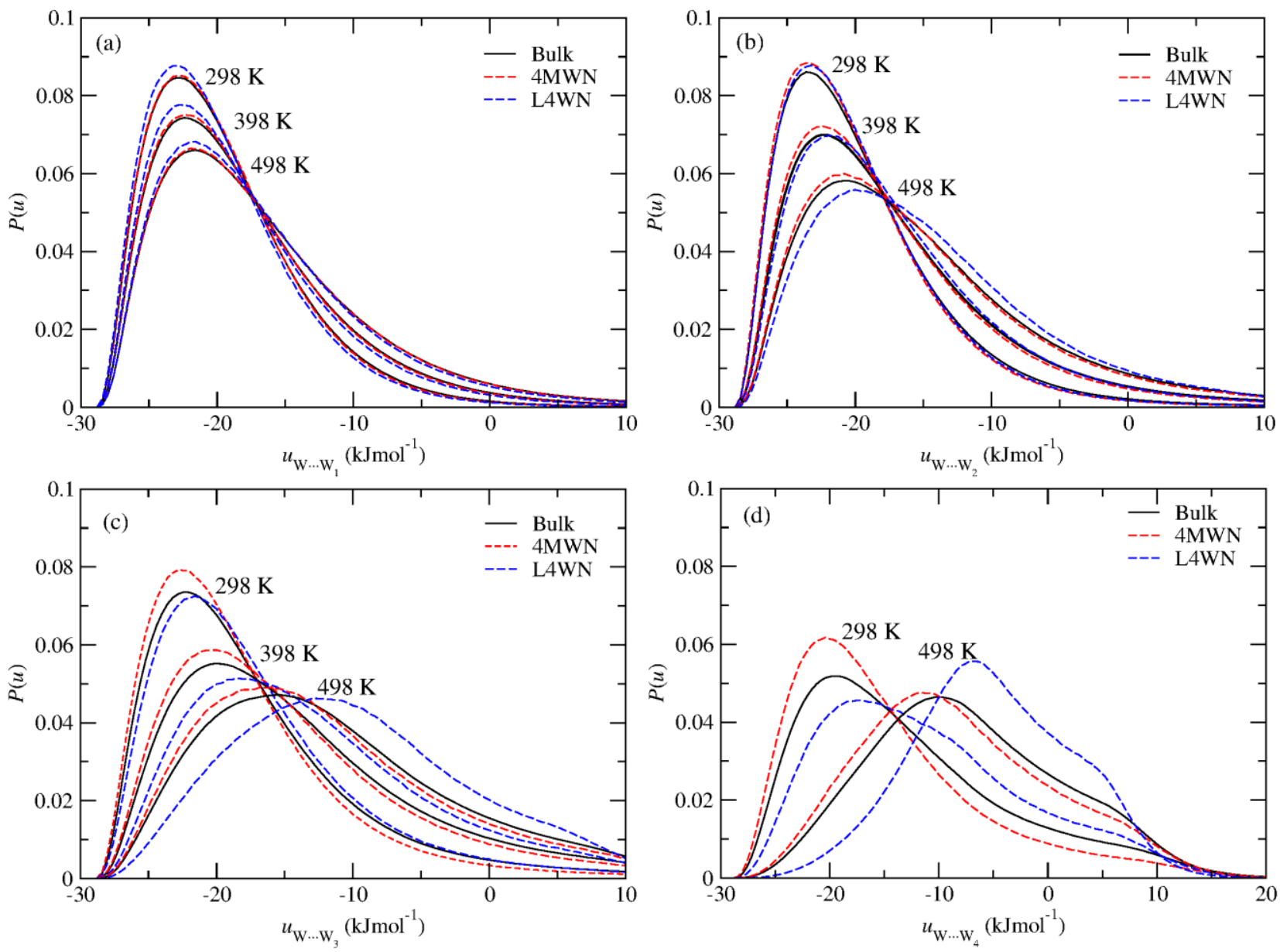

Figure 6 - Temperature dependence of the pair interaction energy distributions for the first, second, third, and fourth nearest water neighbors in the water populations with 4MWN and less than 4 water neighbors (L4WN) in the first hydration shell (HSh) of the methyl groups of neopentane and in bulk water. The distributions at $398 \mathrm{~K}$ for the fourth nearest water neighbor are omitted for clarity.

As can be seen, the temperature affects more dramatically water molecules with L4WN, that is, those nearest to the solute. Furthermore, this population increases with the temperature, along with a mild dehydration of the solutes (see Table S5). The fact that the 4MWN water population decreases at a slightly higher rate with the temperature, for the larger solutes, along with a slightly higher rate of dehydration (see Table S5), is consistent with the larger (positive) $\Delta S_{\text {hyd }}$ of neopentane, relative to methane, above the entropic convergence temperature, $440 \mathrm{~K}$ (see Fig. $\left.1\left(b_{1}\right)\right)$. For the amphiphilic solutes a similar behavior is observed. Entropic convergence occurs, however, at $T<T_{S}$ and a less negative $\Delta S_{\text {hyd }}$ is observed for neopentanol, relative to methanol, above $\sim 460 \mathrm{~K}$ (see Fig. 3(b $\left.\mathrm{b}_{1}\right)$ ). This negative entropy, as opposed to methane and neopentane, results from electrostatic interactions, as previously discussed, which are also responsible for the solute size dependence of $\Delta S_{\text {hyd }} / S A S A$, opposite to hydrocarbons. 
The above results indicate that larger hydrophobic groups induce a more liquid-vapor like interface at every temperature, characterized by more water molecules with dangling $\mathrm{OH}$ groups (L4WN population), and this difference is intensified with the temperature. This solute size dependence, disappears, however, for $\Delta S_{\text {hyd }} / S A S A$, since the latter accounts for the number of water molecules in the hydration shell (see Table S6), thus, normalizing the solute size dependence of $\Delta S_{\text {hyd }}$ with respect to solute-water (van der Waals) and water-water interactions, in the first coordination sphere. Thus, while solute-water interactions greatly influence the temperature dependence of the rate of increase of $\Delta S_{\text {hyd }}$, and, therefore, $T_{S}$, water-water interactions, associated with structural perturbations next to the solutes, also play an important role, contributing to the reversal of the solute-size dependence of $\Delta S_{\text {hyd }}$, above the entropic convergence temperature. This picture is fully supported by the fact that solute-water interactions do not converge at any temperature (see Fig. S6), opposite to $\Delta H_{\text {hyd }}$, thus indicating that water-water interactions should be important to explain the reversal of the solute size dependence of both $\Delta H_{\text {hyd }}$ and $\Delta S_{\text {hyd }}$.

\section{Conclusions}

The hydration of hydrophobic and amphiphilic solutes was studied through molecular dynamics, aiming at gaining insight on the singular behavior of water, concerning entropic convergence and the hydrophobic zero entropy, $T_{S}$, also observed upon protein denaturation. Our results show that the difference between the $T_{S}$ of hydrophobic and amphiphilic solutes is associated with solute-water interactions, namely, electrostatic interactions. Further, the reversal of the solutesize dependence of $\Delta S_{\text {hyd }}$, above the entropic convergence temperature, is closely associated with a higher rate of dehydration of larger solutes, influencing both, solute-water and water-water interactions. Remarkably, the $\Delta S_{\text {hyd }}$, normalized by the SASA, shows a nearly solute size independence, for hydrocarbons, below and above $T_{S}$. For amphiphilic solutes, $\Delta S_{\text {hyd }} / \mathrm{SASA}$, does not exhibit a similar behavior because of long- range electrostatic interactions, much less sensible to the SASA. We also show that the tetrahedral enhancement of water next to hydrophobic groups is already encoded in neat water populations lacking interstitial waters, and that this is lost at high temperatures, both in pure water and next to hydrophobic solutes.

The source of the differences between Kauzmann's "hydrocarbon model" on protein denaturation and hydrophobic hydration, regarding the zero entropy and enthalpy temperatures, is also discussed, with relatively large amphiphilic (e.g. neopentanol) and polycyclic aromatic hydrocarbons displaying a more similar behavior to globular proteins, than aliphatic hydrocarbons 
or small amphiphilic molecules. This is consistent with the fact that the core of globular proteins is not exclusively populated by aliphatic residues.

\section{Acknowledgements}

KT would like to acknowledge an undergraduate ERASMUS scholarship. NG would like to acknowledge financial support from Fundação para a Ciência e a Tecnologia of Portugal (SFRH/BPD/110483/2015). NG would also like to thank Prof. Hank Ashbaugh for discussions on the zero entropy and information theory, and for reading the manuscript. This work was supported by UID/MULTI/04046/2013 centre grant from FCT, Portugal (to BioISI) and by the Portuguese National Distributed Computing Infrastructure (http://www.incd.pt).

\section{Supporting Information}

Various results, discussed in the manuscript, including, the $\Delta G_{\text {hyd }}$ for different water models at 298 $\mathrm{K}$ and $1 \mathrm{~atm}$ (Table S1), SASA for distinct molecules (Table S2), parameters for the generalized equation of $\Delta S_{\text {hyd }} / S A S A$ (Table S3), average tetrahedrality of different water populations at $298 \mathrm{~K}$ and $498 \mathrm{~K}$ (Table S4), $T$ dependence of the coordination numbers of different water populations (Tables S5 and S6), the $T$ dependence of the thermodynamic functions for methane and methanol at 1 atm (Fig. S1), the $T$ dependence of the solute-water interaction energy for aliphatic hydrocarbons (Fig. S2), $T$ dependence of $\Delta S_{\text {hyd }} / S A S A$ for hydrocarbons (Fig. S3), the $T$ dependence of the solutewater interaction energy for aromatic hydrocarbons (Fig. S4) and amphiphilic solutes (Fig. S5), and a plot of the $T$ dependence of the solute-water energy of hydrocarbons and amphiphilic solutes (Fig. S6), are available, free of charge in the Supporting Information.

\section{References}

1. W. Blokzijl, J. B. F. N. Engberts, Hydrophobic Effects. Opinions and Facts. Angew. Chem. Int. Ed. Engl. 32, 1545-1579 (1993).

2. D. Chandler, Interfaces and the driving force of hydrophobic assembly. Nature 437, 640-647 (2005).

3. H. S. Ashbaugh, L. R. Pratt, Colloquium: Scaled particle theory and the length scales of hydrophobicity. Rev. Mod. Phys. 78, 159-178 (2006).

4. N. T. Southall, K. A. Dill, A. D. J. Haymet, A View of the Hydrophobic Effect. J. Phys. Chem. $B$ 106, 521-533 (2002).

5. P. L. Privalov, S. J. Gill, The hydrophobic effect: a reappraisal. Pure Appl. Chem. 61, 10971104 (1989). 
6. G. I. Makhatadze, P. L. Privalov, Hydration effects in protein unfolding. Biophys. Chem. 51, 291-309 (1994).

7. R. L. Baldwin, Temperature dependence of the hydrophobic interaction in protein folding. Proc. Natl. Acad. Sci. 83, 8069-8072 (1986).

8. S. Garde, G. Hummer, A. E. García, M. E. Paulaitis, L. R. Pratt, Origin of Entropy Convergence in Hydrophobic Hydration and Protein Folding. Phys. Rev. Lett. 77, 4966-4968 (1996).

9. P. L. Privalov, S. J. Gill, "Stability of Protein Structure and Hydrophobic Interaction" in Advances in Protein Chemistry, (Elsevier, 1988), pp. 191-234.

10. P. L. Privalov, "Stability of Proteins Small Globular Proteins" in Advances in Protein Chemistry, (Elsevier, 1979), pp. 167-241.

11. W. Kauzmann, Some factors in the interpretation of protein denaturation. Adv. Protein Chem. 14, 1-63 (1959).

12. R. L. Baldwin, G. D. Rose, How the hydrophobic factor drives protein folding. Proc. Natl. Acad. Sci. 113, 12462-12466 (2016).

13. K. Murphy, P. Privalov, S. Gill, Common features of protein unfolding and dissolution of hydrophobic compounds. Science 247, 559-561 (1990).

14. D. M. Huang, D. Chandler, Temperature and length scale dependence of hydrophobic effects and their possible implications for protein folding. Proc. Natl. Acad. Sci. U. S. A. 97, 83248327 (2000).

15. A. Ben-Naim, Molecular Theory of Water and Aqueous Solutions: Part II: The Role of Water in Protein Folding, Self-Assembly and Molecular Recognition (World Scientific Publishing Company, 2011) https:/doi.org/10.1142/8154 (November 12, 2019).

16. R. L. Baldwin, Gas-liquid transfer data used to analyze hydrophobic hydration and find the nature of the Kauzmann-Tanford hydrophobic factor. Proc. Natl. Acad. Sci. 109, 7310-7313 (2012).

17. N. Galamba, A. Paiva, S. Barreiros, P. Simões, Solubility of Polar and Nonpolar Aromatic Molecules in Subcritical Water: The Role of the Dielectric Constant. J. Chem. Theory Comput. 15, 6277-6293 (2019).

18. R. L. Baldwin, The new view of hydrophobic free energy. FEBS Lett. 587, 1062-1066 (2013).

19. B. Lee, Solvent reorganization contribution to the transfer thermodynamics of small nonpolar molecules. Biopolymers 31, 993-1008 (1991).

20. T. Lazaridis, Solvent Size vs Cohesive Energy as the Origin of Hydrophobicity. Acc. Chem. Res. 34, 931-937 (2001).

21. R. L. Baldwin, Dynamic hydration shell restores Kauzmann's 1959 explanation of how the hydrophobic factor drives protein folding. Proc. Natl. Acad. Sci. U. S. A. 111, 13052-13056 (2014).

22. B. Lee, The physical origin of the low solubility of nonpolar solutes in water. Biopolymers $\mathbf{2 4}$, 
813-823 (1985).

23. L. R. Pratt, A. Pohorille, Theory of hydrophobicity: Transient cavities in molecular liquids. Proc. Natl. Acad. Sci. 89, 2995-2999 (1992).

24. A. Ben-Naim, Hydrophobic interaction and structural changes in the solvent. Biopolymers 14, 1337-1355 (1975).

25. H.-A. Yu, M. Karplus, A thermodynamic analysis of solvation. J. Chem. Phys. 89, 2366 (1988).

26. E. Grunwald, C. Steel, Solvent Reorganization and Thermodynamic Enthalpy-Entropy Compensation. J. Am. Chem. Soc. 117, 5687-5692 (1995).

27. H. Qian, J. J. Hopfield, Entropy-enthalpy compensation: Perturbation and relaxation in thermodynamic systems. J. Chem. Phys. 105, 9292 (1996).

28. D. Ben-Amotz, R. Underwood, Unraveling Water's Entropic Mysteries: A Unified View of Nonpolar, Polar, and Ionic Hydration. Acc. Chem. Res. 41, 957-967 (2008).

29. A. Ben-Naim, A simple model for demonstrating the relation between solubility, hydrophobic interaction, and structural changes in the solvent. J. Phys. Chem. 82, 874-885 (1978).

30. J. Turner, A. K. Soper, The effect of apolar solutes on water structure: Alcohols and tetraalkylammonium ions. J. Chem. Phys. 101, 6116 (1994).

31. A. K. Soper, J. L. Finney, Hydration of methanol in aqueous solution. Phys. Rev. Lett. 71, 4346-4349 (1993).

32. P. Buchanan, N. Aldiwan, A. K. Soper, J. L. Creek, C. A. Koh, Decreased structure on dissolving methane in water. Chem. Phys. Lett. 415, 89-93 (2005).

33. M. P. S. Mateus, N. Galamba, B. J. C. Cabral, Structure and electronic properties of a benzenewater solution. J. Chem. Phys. 136, 014507 (2012).

34. N. Galamba, Water's Structure around Hydrophobic Solutes and the Iceberg Model. J. Phys. Chem. B 117, 2153-2159 (2013).

35. N. Galamba, Reply to "Comment on 'Water's Structure around Hydrophobic Solutes and the Iceberg Model.”' J. Phys. Chem. B 118, 2600-2603 (2014).

36. N. Galamba, Water Tetrahedrons, Hydrogen-Bond Dynamics, and the Orientational Mobility of Water around Hydrophobic Solutes. J. Phys. Chem. B 118, 4169-4176 (2014).

37. H. F. M. C. Martiniano, N. Galamba, Fast and slow dynamics and the local structure of liquid and supercooled water next to a hydrophobic amino acid. Phys Chem Chem Phys 18, 2763927647 (2016).

38. B. Song, V. Molinero, Thermodynamic and structural signatures of water-driven methanemethane attraction in coarse-grained $\mathrm{mW}$ water. J. Chem. Phys. 139, 054511 (2013).

39. H. S. Ashbaugh, J. W. Barnett, A. Saltzman, M. E. Langrehr, H. Houser, Communication: Stiffening of dilute alcohol and alkane mixtures with water. J. Chem. Phys. 145, 201102 (2016). 
40. S. Daschakraborty, How do glycerol and dimethyl sulphoxide affect local tetrahedral structure of water around a nonpolar solute at low temperature? Importance of preferential interaction. J. Chem. Phys. 148, 134501 (2018).

41. F. Merzel, F. Avbelj, Why do water molecules around small hydrophobic solutes form stronger hydrogen bonds than in the bulk? Biochim. Biophys. Acta BBA - Gen. Subj. 1864, 129537 (2020).

42. J. G. Davis, K. P. Gierszal, P. Wang, D. Ben-Amotz, Water structural transformation at molecular hydrophobic interfaces. Nature 491, 582-585 (2012).

43. X. Wu, W. Lu, L. M. Streacker, H. S. Ashbaugh, D. Ben-Amotz, Methane Hydration-Shell Structure and Fragility. Angew. Chem. Int. Ed. 57, 15133-15137 (2018).

44. J. Grdadolnik, F. Merzel, F. Avbelj, Origin of hydrophobicity and enhanced water hydrogen bond strength near purely hydrophobic solutes. Proc. Natl. Acad. Sci. 114, 322-327 (2017).

45. G.-H. Deng, et al., Ordered-to-Disordered Transformation of Enhanced Water Structure on Hydrophobic Surfaces in Concentrated Alcohol-Water Solutions. J. Phys. Chem. Lett. 10, 7922-7928 (2019).

46. N. Galamba, On the hydrogen-bond network and the non-Arrhenius transport properties of water. J. Phys. Condens. Matter 29, 015101 (2017).

47. F. H. Stillinger, Structure in aqueous solutions of nonpolar solutes from the standpoint of scaled-particle theory. J. Solut. Chem. 2, 141-158 (1973).

48. L. R. Pratt, D. Chandler, Theory of the hydrophobic effect. J. Chem. Phys. 67, 3683 (1977).

49. G. Hummer, S. Garde, A. E. Garcia, A. Pohorille, L. R. Pratt, An information theory model of hydrophobic interactions. Proc. Natl. Acad. Sci. 93, 8951 (1996).

50. B. J. Berne, Inferring the hydrophobic interaction from the properties of neat water. Proc. Natl. Acad. Sci. 93, 8800-8803 (1996).

51. G. Graziano, Contrasting the hydration thermodynamics of methane and methanol. Phys. Chem. Chem. Phys. 21, 21418-21430 (2019).

52. W. L. Jorgensen, D. S. Maxwell, J. Tirado-Rives, Development and Testing of the OPLS AllAtom Force Field on Conformational Energetics and Properties of Organic Liquids. J. Am. Chem. Soc. 118, 11225-11236 (1996).

53. D. Van Der Spoel, et al., GROMACS: fast, flexible, and free. J. Comput. Chem. 26, 17011718 (2005).

54. W. L. Jorgensen, J. Chandrasekhar, J. D. Madura, R. W. Impey, M. L. Klein, Comparison of simple potential functions for simulating liquid water. J. Chem. Phys. 79, 926-935 (1983).

55. H. J. C. Berendsen, J. R. Grigera, T. P. Straatsma, The missing term in effective pair potentials. J. Phys. Chem. 91, 6269-6271 (1987).

56. H. W. Horn, et al., Development of an improved four-site water model for biomolecular simulations: TIP4P-Ew. J. Chem. Phys. 120, 9665-9678 (2004). 
57. J. L. F. Abascal, C. Vega, A general purpose model for the condensed phases of water: TIP4P/2005. J. Chem. Phys. 123, 234505 (2005).

58. G. Bussi, D. Donadio, M. Parrinello, Canonical sampling through velocity rescaling. J. Chem. Phys. 126, 014101 (2007).

59. M. Parrinello, Polymorphic transitions in single crystals: A new molecular dynamics method. J. Appl. Phys. 52, 7182 (1981).

60. U. Essmann, et al., A smooth particle mesh Ewald method. J. Chem. Phys. 103, 8577 (1995).

61. B. Hess, H. Bekker, H. J. C. Berendsen, J. G. E. M. Fraaije, LINCS: A linear constraint solver for molecular simulations. J. Comput. Chem. 18, 1463-1472 (1997).

62. G. Duarte Ramos Matos, et al., Approaches for Calculating Solvation Free Energies and Enthalpies Demonstrated with an Update of the FreeSolv Database. J. Chem. Eng. Data 62, 1559-1569 (2017).

63. C. H. Bennett, Efficient estimation of free energy differences from Monte Carlo data. $J$. Comput. Phys. 22, 245-268 (1976).

64. A. Ben-Naim, Y. Marcus, Solvation thermodynamics of nonionic solutes. J. Chem. Phys. 81, 2016-2027 (1984).

65. W. F. Van Gunsteren, H. J. C. Berendsen, A Leap-frog Algorithm for Stochastic Dynamics. Mol. Simul. 1, 173-185 (1988).

66. T. Steinbrecher, I. Joung, D. A. Case, Soft-core potentials in thermodynamic integration: Comparing one- and two-step transformations. J. Comput. Chem. 32, 3253-3263 (2011).

67. T. C. Beutler, A. E. Mark, R. C. van Schaik, P. R. Gerber, W. F. van Gunsteren, Avoiding singularities and numerical instabilities in free energy calculations based on molecular simulations. Chem. Phys. Lett. 222, 529-539 (1994).

68. V. Gapsys, D. Seeliger, B. L. de Groot, New Soft-Core Potential Function for Molecular Dynamics Based Alchemical Free Energy Calculations. J. Chem. Theory Comput. 8, 23732382 (2012).

69. T. T. Pham, M. R. Shirts, Identifying low variance pathways for free energy calculations of molecular transformations in solution phase. J. Chem. Phys. 135, 034114 (2011).

70. M. Parrinello, Polymorphic transitions in single crystals: A new molecular dynamics method. J. Appl. Phys. 52, 7182 (1981).

71. E. Gallicchio, M. M. Kubo, R. M. Levy, Enthalpy-Entropy and Cavity Decomposition of Alkane Hydration Free Energies: Numerical Results and Implications for Theories of Hydrophobic Solvation. J. Phys. Chem. B 104, 6271-6285 (2000).

72. A. Shrake, J. A. Rupley, Environment and exposure to solvent of protein atoms. Lysozyme and insulin. J. Mol. Biol. 79, 351-371 (1973).

73. F. Eisenhaber, P. Lijnzaad, P. Argos, C. Sander, M. Scharf, The double cubic lattice method: Efficient approaches to numerical integration of surface area and volume and to dot surface contouring of molecular assemblies. J. Comput. Chem. 16, 273-284 (1995). 
74. A. Bondi, van der Waals Volumes and Radii. J. Phys. Chem. 68, 441-451 (1964).

75. P.-L. Chau, A. J. Hardwick, A new order parameter for tetrahedral configurations. Mol. Phys. 93, 511-518 (1998).

76. J. R. Errington, P. G. Debenedetti, Relationship between structural order and the anomalies of liquid water. Nature 409, 318-321 (2001).

77. A. Ben-Naim, M. Yaacobi, Effects of Solutes on the Strength of Hydrophobic Interaction and Its Temperature Dependence. J. Phys. Chem. 78, 170-175 (1974).

78. H. Naghibi, S. F. Dec, S. J. Gill, Heat of solution of methane in water from 0 to 50.degree.C. J. Phys. Chem. 90, 4621-4623 (1986).

79. J. Wang, R. M. Wolf, J. W. Caldwell, P. A. Kollman, D. A. Case, Development and testing of a general amber force field. J. Comput. Chem. 25, 1157-1174 (2004).

80. B. Widom, Potential-distribution theory and the statistical mechanics of fluids. J. Phys. Chem. 86, 869-872 (1982).

81. S. Cabani, P. Gianni, V. Mollica, L. Lepori, Group contributions to the thermodynamic properties of non-ionic organic solutes in dilute aqueous solution. J. Solut. Chem. 10, 563-595 (1981).

82. G. I. Makhatadze, P. L. Privalov, Heat capacity of alcohols in aqueous solutions in the temperature range from 5 to 125C. J. Solut. Chem. 18, 927-936 (1989).

83. J. A. Schellman, Temperature, stability, and the hydrophobic interaction. Biophys. J. 73, 29602964 (1997).

84. N. Galamba, B. J. C. Cabral, The Changing Hydrogen-Bond Network of Water from the Bulk to the Surface of a Cluster: A Born-Oppenheimer Molecular Dynamics Study. J. Am. Chem. Soc. 130, 17955-17960 (2008).

85. Q. Du, R. Superfine, E. Freysz, Y. R. Shen, Vibrational spectroscopy of water at the vapor/water interface. Phys. Rev. Lett. 70, 2313-2316 (1993).

86. E. A. Raymond, T. L. Tarbuck, M. G. Brown, G. L. Richmond, Hydrogen-Bonding Interactions at the Vapor/Water Interface Investigated by Vibrational Sum-Frequency Spectroscopy of HOD/H ${ }_{2} \mathrm{O} / \mathrm{D}_{2} \mathrm{O}$ Mixtures and Molecular Dynamics Simulations. J. Phys. Chem. B 107, 546-556 (2003).

87. I. Benjamin, Vibrational Spectrum of Water at the Liquid/Vapor Interface. Phys. Rev. Lett. 73, 2083-2086 (1994).

88. E. Shiratani, M. Sasai, Growth and collapse of structural patterns in the hydrogen bond network in liquid water. J. Chem. Phys. 104, 7671-7680 (1996). 


\section{Protein Denaturation, Zero Entropy Temperature, and the Structure of Water around Hydrophobic and Amphiphilic Solutes}

Supporting Information

Kazimieras Tamoliūnas ${ }^{\mathrm{a}}$, Nuno Galamba ${ }^{\mathrm{a}, *}$

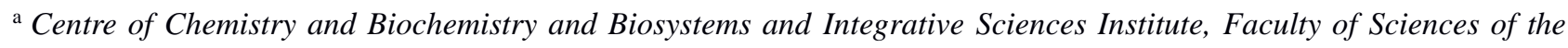
University of Lisbon, C8, Campo Grande, 1749-016 Lisbon, Portugal.

${ }^{*}$ Corresponding author. Electronic mail: njgalamba@fc.ul.pt 
Table S1 - Hydration free energy at $298 \mathrm{~K}$ and 1 atm for various nonpolar and polar OPLS-aa model solutes in different water models.

\begin{tabular}{cccccc}
\hline & TIP3P & SPC/E & TIP4P-Ew & TIP4P-2005 & Experimental \\
& $\Delta G_{\mathrm{hyd}}\left(\mathrm{kJmol}^{-1}\right)$ & $\Delta G_{\mathrm{hyd}}\left(\mathrm{kJmol}^{-1}\right)$ & $\Delta G_{\mathrm{hyd}}\left(\mathrm{kJmol}^{-1}\right)$ & $\Delta G_{\mathrm{hyd}}\left(\mathrm{kJmol}^{-1}\right)$ & $\Delta G_{\mathrm{hyd}}\left(\mathrm{kJmol}^{-1}\right)$ \\
\hline Methane & $+9.1 \pm 0.02$ & $+9.5 \pm 0.1$ & $+9.5 \pm 0.02$ & $+9.4 \pm 0.04$ & +8.4 \\
Methanol & $-17.8 \pm 0.1$ & $-18.0 \pm 0.1$ & $-18.4 \pm 0.1$ & $-18.9 \pm 0.15$ & -21.3 \\
Benzene & $-1.5 \pm 0.1$ & $-0.2 \pm 0.1$ & $+0.1 \pm 0.1$ & $-0.6 \pm 0.1$ & -3.6 \\
Phenol & $-21.7 \pm 0.02$ & $-20.1 \pm 0.2$ & $-19.7 \pm 0.1$ & $-20.2 \pm 0.1$ & -27.7 \\
Naphthalene & $-7.8 \pm 0.1$ & $-5.5 \pm 0.1$ & $-5.0 \pm 0.1$ & $-5.9 \pm 0.2$ & -9.6 \\
Anthracene & $-13.2 \pm 0.2$ & $-10.1 \pm 0.1$ & $-9.2 \pm 0.1$ & $-10.7 \pm 0.1$ & -17.7 \\
\hline
\end{tabular}

Table S2 - Solvent accessible surface area (SASA) values for the different solutes.

\begin{tabular}{cccccc}
\hline Solute & SASA $^{1}\left(\mathrm{~nm}^{2}\right)$ & Solute & SASA $\left(\mathrm{nm}^{2}\right)$ & Solute & SASA $\left(\mathrm{nm}^{2}\right)$ \\
\hline Methane & $1.43(6)$ & Methanol & $1.63(0)$ & Benzene & $2.44(1)$ \\
Ethane & $1.81(7)$ & Ethanol & $1.97(9)$ & Naphthalene & $3.10(3)$ \\
Neopentane & $2.60(7)$ & Neopentanol & $2.58(3)$ & Anthracene & $3.76(4)$ \\
Pentane & $2.75(0)$ & Phenol & $2.70(5)$ & Pyrene & $3.87(3)$ \\
Heptane & $3.36(3)$ & & & & \\
\hline
\end{tabular}

${ }^{1}$ Solvent sphere radius $1.4 \AA$.

Table S3 - Generalized parameters for the temperature dependence of $T \Delta S_{\mathrm{hyd}}\left(\mathrm{kJmol}^{-1}\right)$, normalized by the SASA $\left(\mathrm{nm}^{2}\right)$, for aliphatic and aromatic hydrocarbons. The equation is of the form $T \triangle S / S A S A=A+B T+C T^{2}$, where $T$ is the temperature in $\mathrm{K}$.

\begin{tabular}{cccc}
\hline & $\mathrm{A}$ & $\mathrm{B}$ & $\mathrm{C}$ \\
\hline Aliphatic & 0.0185382 & -0.123008 & 0.000278762 \\
Aromatic & 0.00437478 & -0.100095 & 0.000211814 \\
\hline
\end{tabular}

Table S4 - Mean tetrahedrality, $\langle q\rangle$, of different water populations at $298 \mathrm{~K}$ and $498 \mathrm{~K}$. The no interstitial water molecules (NIWN) population includes water molecules surrounded by no more than 4 water neighbors up to $3.7 \AA$. The interstitial water molecules (IWN) population includes water molecules surrounded by 5 water neighbors up to $3.7 \AA$. The 4 or more water neighbors (4MWN) population includes water molecules in the first coordination sphere of neopentane that retain four or more water neighbors, closer than any atom of the solute.

\begin{tabular}{ccc}
\hline & \multicolumn{2}{c}{$\langle q\rangle$} \\
\hline Water Population & $298 \mathrm{~K}$ & $498 \mathrm{~K}$ \\
\hline Bulk & 0.668 & 0.477 \\
Bulk - NIWM & 0.717 & 0.443 \\
Bulk - IWM & 0.662 & 0.489 \\
Neopentane - 4MWN & 0.697 & 0.476 \\
\hline
\end{tabular}


Table S5 - Temperature dependence of the number of water molecules in the first hydration shell, $N_{\mathrm{HSh}}$, and the respective number of water molecules with four or more water neighbors (4MWN), $N_{4 \mathrm{MWN}}$, for different solutes.

\begin{tabular}{ccccc}
\hline & $298 \mathrm{~K}$ & $398 \mathrm{~K}$ & $498 \mathrm{~K}$ & $\Delta(498 \mathrm{~K}-298 \mathrm{~K})$ \\
& $N_{\mathrm{HSh}}\left[N_{4 \mathrm{MWN}}\right]$ & $N_{\mathrm{HSh}}\left[N_{4 \mathrm{MWN}}\right]$ & $N_{\mathrm{HSh}}\left[N_{4 \mathrm{MWN}}\right]$ & $\Delta N_{\mathrm{HSh}}\left[\Delta N_{4 \mathrm{MWN}}\right]$ \\
\hline Methane & $20.3[14.5]$ & $18.7[12.5]$ & $15.8[9.9]$ & $4.5[4.6]$ \\
Methanol & $20.3[13.3]$ & $18.7[11.3]$ & $16.2[9.0]$ & $4.1[4.3]$ \\
Neopentane & $25.9[14.2]$ & $22.2[10.4]$ & $17.7[7.0]$ & $8.2[7.2]$ \\
Neopentanol & $25.4[13.0]$ & $22.3[9.7]$ & $18.2[6.6]$ & $7.2[6.4]$ \\
\hline
\end{tabular}

Table S6 - Temperature dependence of the number of water molecules in the first hydration shell, $N_{\mathrm{HSh}}$, and the respective number of water molecules with four or more water neighbors $(4 \mathrm{MWN}), N_{4 \mathrm{MwN}}$, normalized by the SASA, for different solutes.

\begin{tabular}{ccccc}
\hline & $298 \mathrm{~K}$ & $398 \mathrm{~K}$ & $498 \mathrm{~K}$ & $\Delta(498 \mathrm{~K}-298 \mathrm{~K})$ \\
& $N_{\mathrm{HSh}} / \mathrm{SASA}$ & $N_{\mathrm{HSh}} / \mathrm{SASA}$ & $N_{\mathrm{HSh}} / \mathrm{SASA}$ & $N_{\mathrm{HSh}} / \mathrm{SASA}$ \\
{$\left[N_{4 \mathrm{MWN}} / \mathrm{SASA}\right]$} & {$\left[N_{4 \mathrm{MWN}} / \mathrm{SASA}\right]$} & {$\left[N_{4 \mathrm{MWN}} / \mathrm{SASA}\right]$} & {$\left[N_{4 \mathrm{MWN}} / \mathrm{SASA}\right]$} \\
\hline Methane & $14.1[10.1]$ & $13.0[8.7]$ & $11.0[6.9]$ & $3.1[3.2]$ \\
Methanol & $12.5[8.2]$ & $11.5[6.9]$ & $9.9[5.5]$ & $2.5[2.6]$ \\
Neopentane & $9.9[5.4]$ & $8.5[4.0]$ & $6.8[2.7]$ & $3.1[2.8]$ \\
Neopentanol & $9.4[4.8]$ & $8.2[3.6]$ & $6.7[2.4]$ & $2.7[2.4]$ \\
\hline
\end{tabular}



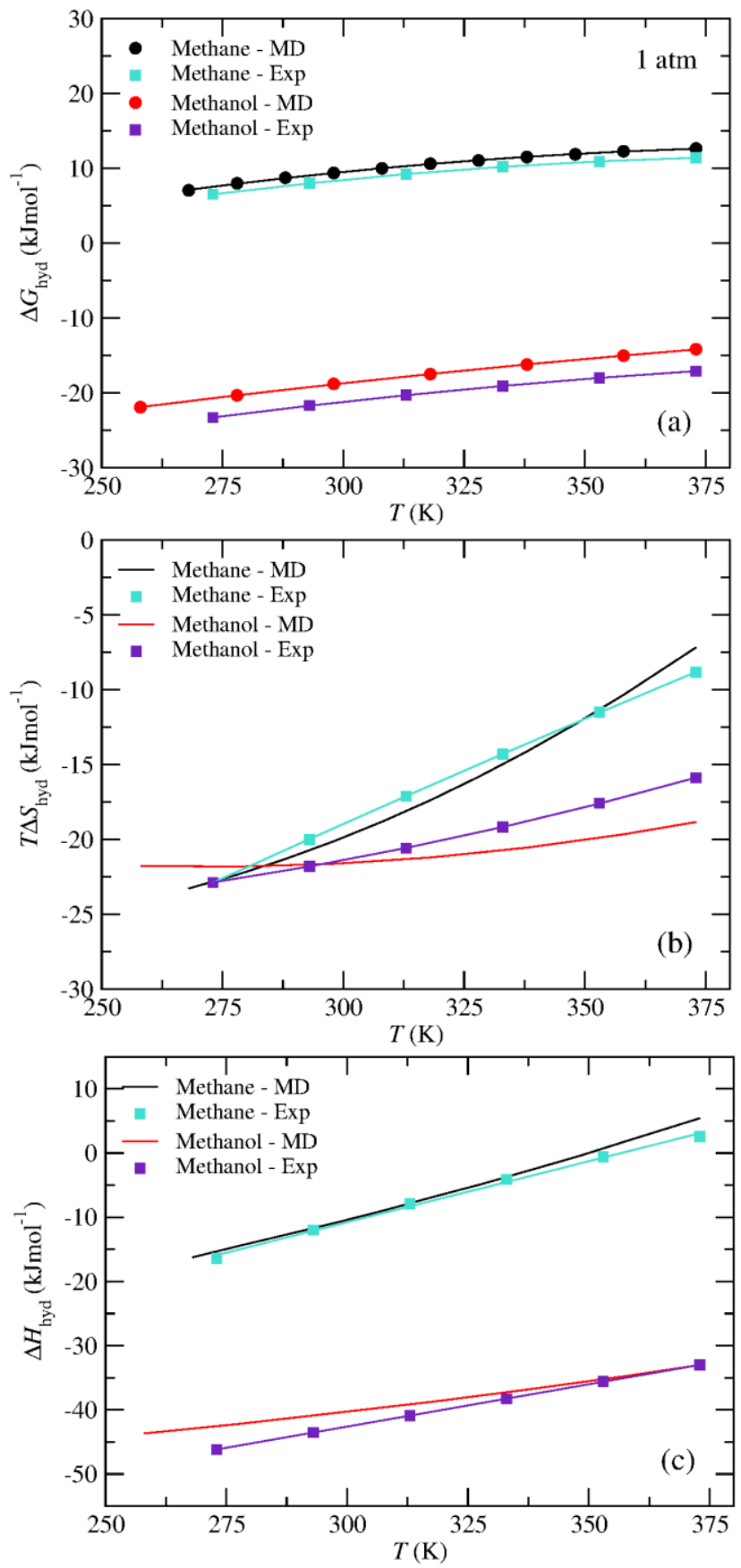

Figure S1 - Temperature dependence of the hydration (a) free energy, (b) entropy, and (c) enthalpy of methane and methanol at $1 \mathrm{~atm}$. The values from molecular dynamics (MD) simulations are compared with available "experimental" data (Table 1 of ref. 51). The methane experimental thermodynamic parameters were obtained from the experimental values at $298 \mathrm{~K}$ and $1 \mathrm{~atm}$ and the experimental $\Delta C_{p}$ up to $323 \mathrm{~K}$ and linear extrapolation of $\Delta C_{p}$ up to $373 \mathrm{~K}$. The heat capacities for methanol used to estimate the thermodynamic parameters were obtained using group additivity contributions. 

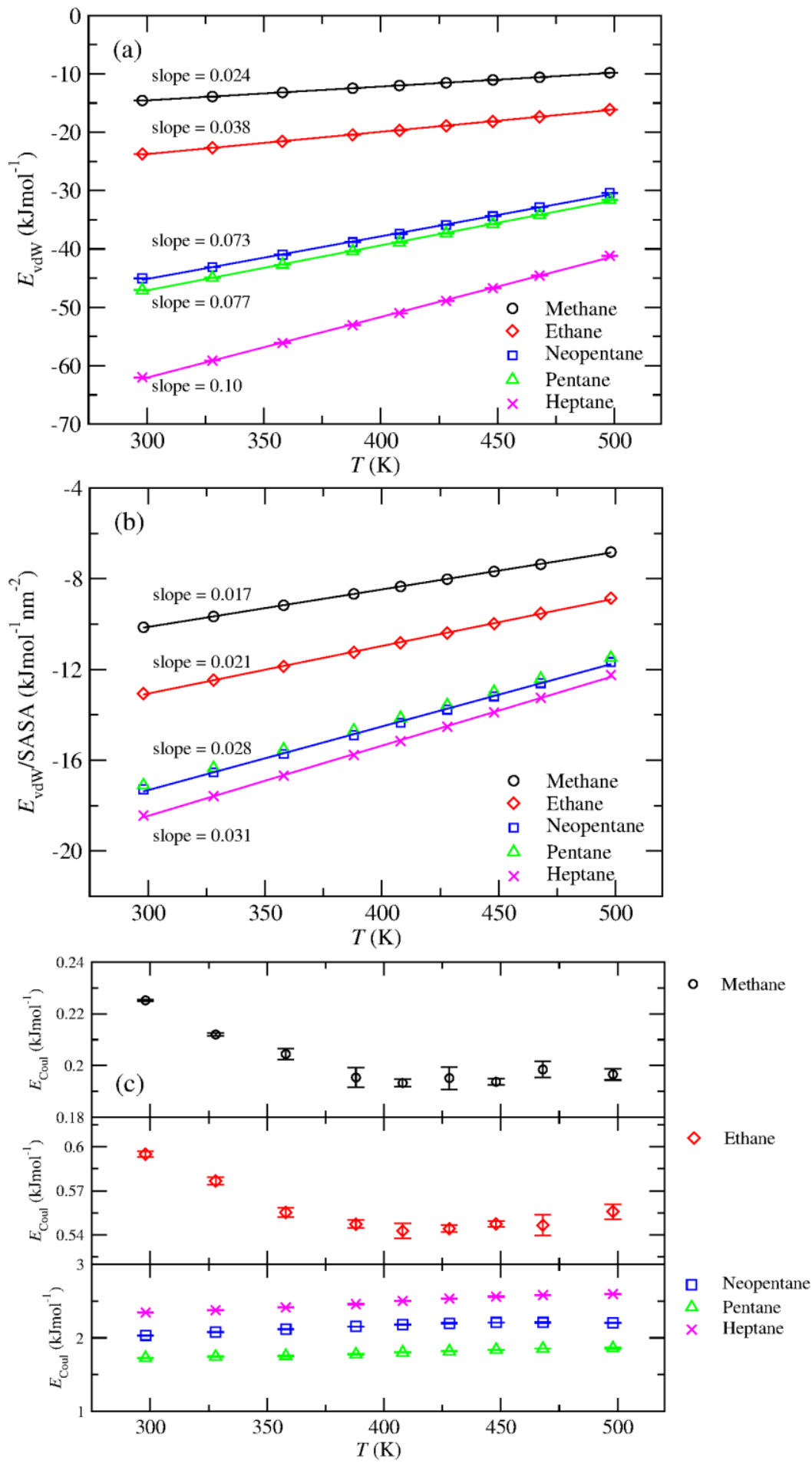

Figure S2 - Temperature dependence of the solute-water (a) van der Waals (vdW) interaction energy, (b) $\mathrm{vdW}$ interaction energy normalized by the SASA, and (c) short range $(r<10 \AA)$ component of the Coulomb interaction energy for the distinct aliphatic hydrocarbons. The rate of increase of the vdW energy with the temperature increases with the solute size. A more similar rate of increase can be observed for the van der Waals energy normalized by the SASA. The long-range contribution of the electrostatic energy is not included because the PME reciprocal space component of the electrostatic potential energy cannot be separated into solute-water and water-water components. The slopes from linear fittings (solid lines) are shown. Errors bars are standard deviations calculated from four, $20 \mathrm{~ns}$ long, independent simulations. 


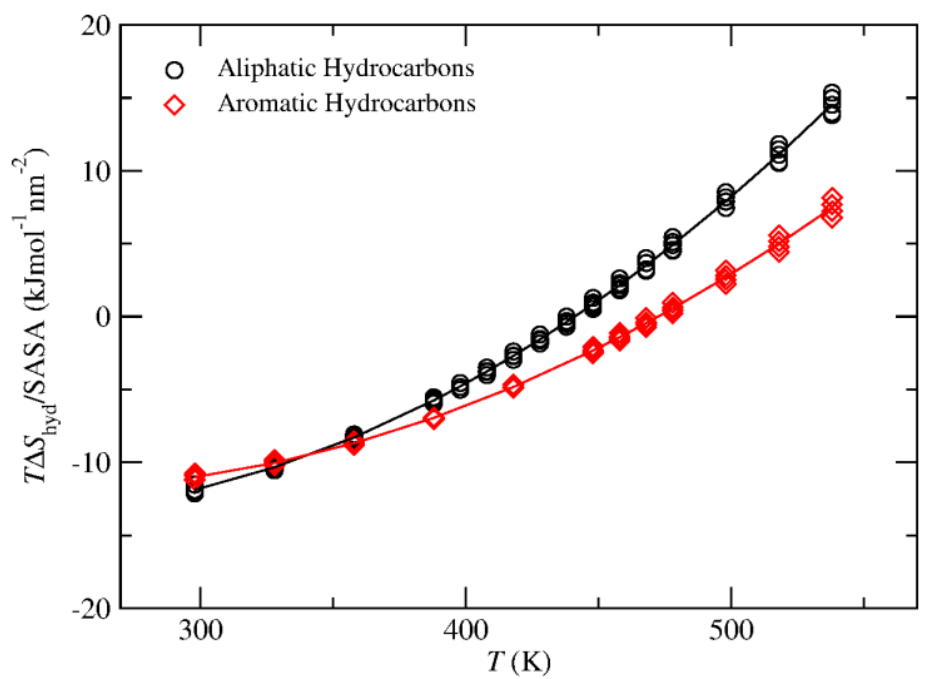

Figure S3 - Generalized (every solute) equations (solid lines) for the hydration entropy of aliphatic and aromatic hydrocarbons, normalized by the SASA. The data was fitted to a second order polynomial, $T \triangle S / S A S A=A+B T+C T^{2}$; parameters are given in Table S3. 

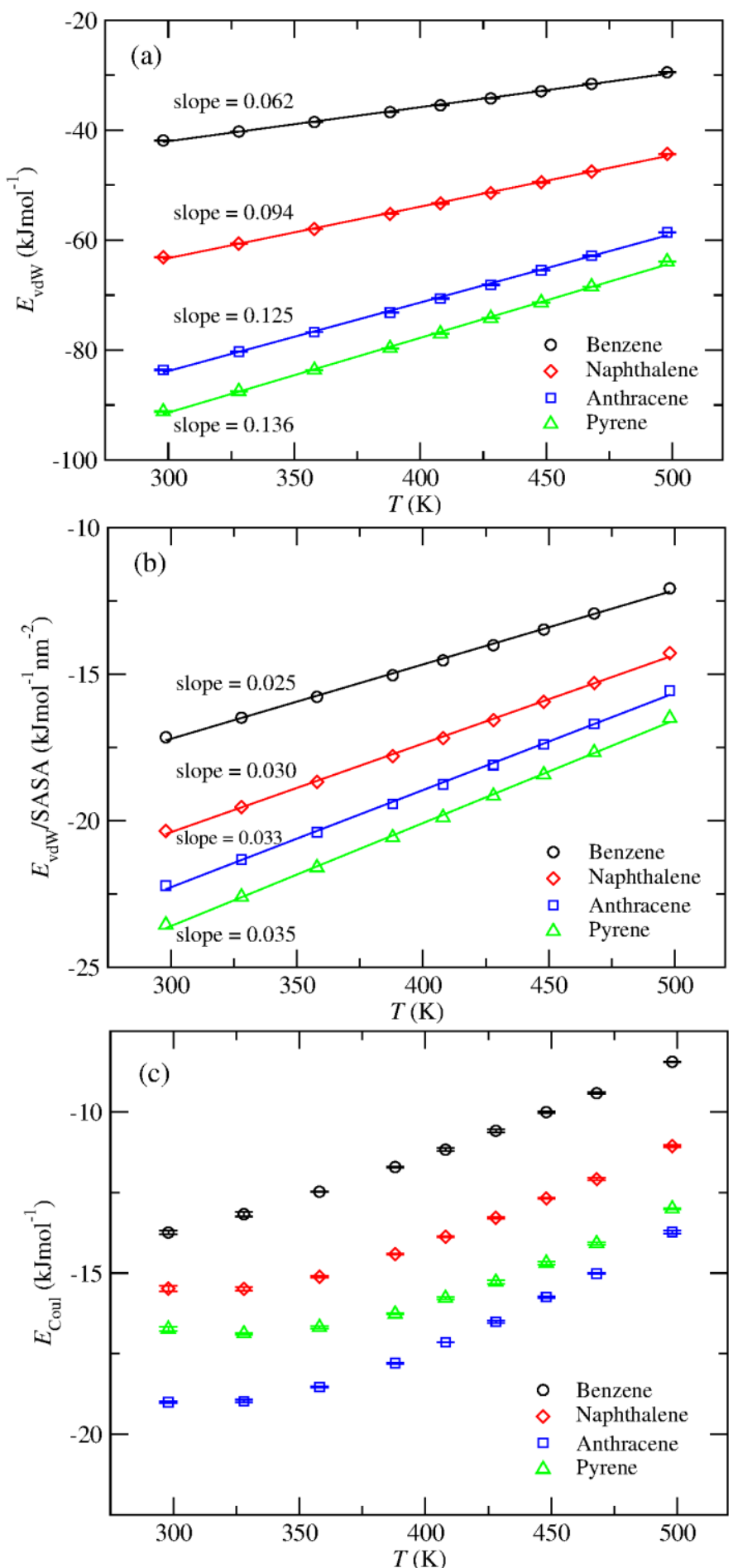

Figure S4 - Temperature dependence of the solute-water (a) van der Waals (vdW) interaction energy, (b) $\mathrm{vdW}$ interaction energy normalized by the SASA, and (c) short range $(r<10 \AA)$ component of the Coulomb interaction energy for the distinct aromatic hydrocarbons. The rate of increase of the vdW energy with the temperature increases with the solute size. A more similar rate of increase can be observed for the van der Waals energy normalized by the SASA. The slopes from linear fittings (solid lines) are shown. Errors bars are standard deviations calculated from four, $20 \mathrm{~ns}$ long, independent simulations. 

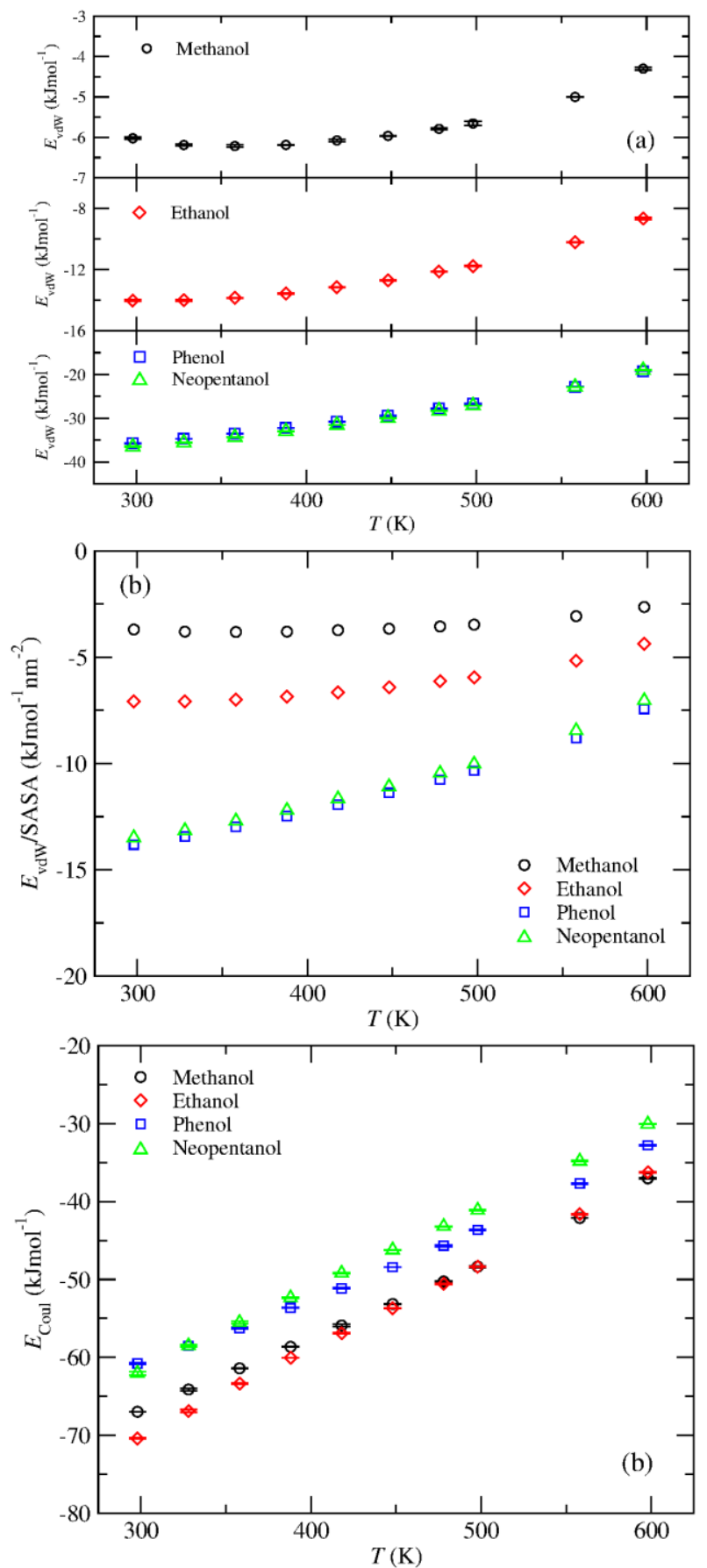

Figure S5 - Temperature dependence of the solute-water (a) van der Waals (vdW) interaction energy, (b) $\mathrm{vdW}$ interaction energy normalized by the SASA, and (c) short range $(r<10 \AA)$ component of the Coulomb interaction energy for the distinct alcohols. Errors bars are standard deviations calculated from four, $20 \mathrm{~ns}$ long, independent simulations. 

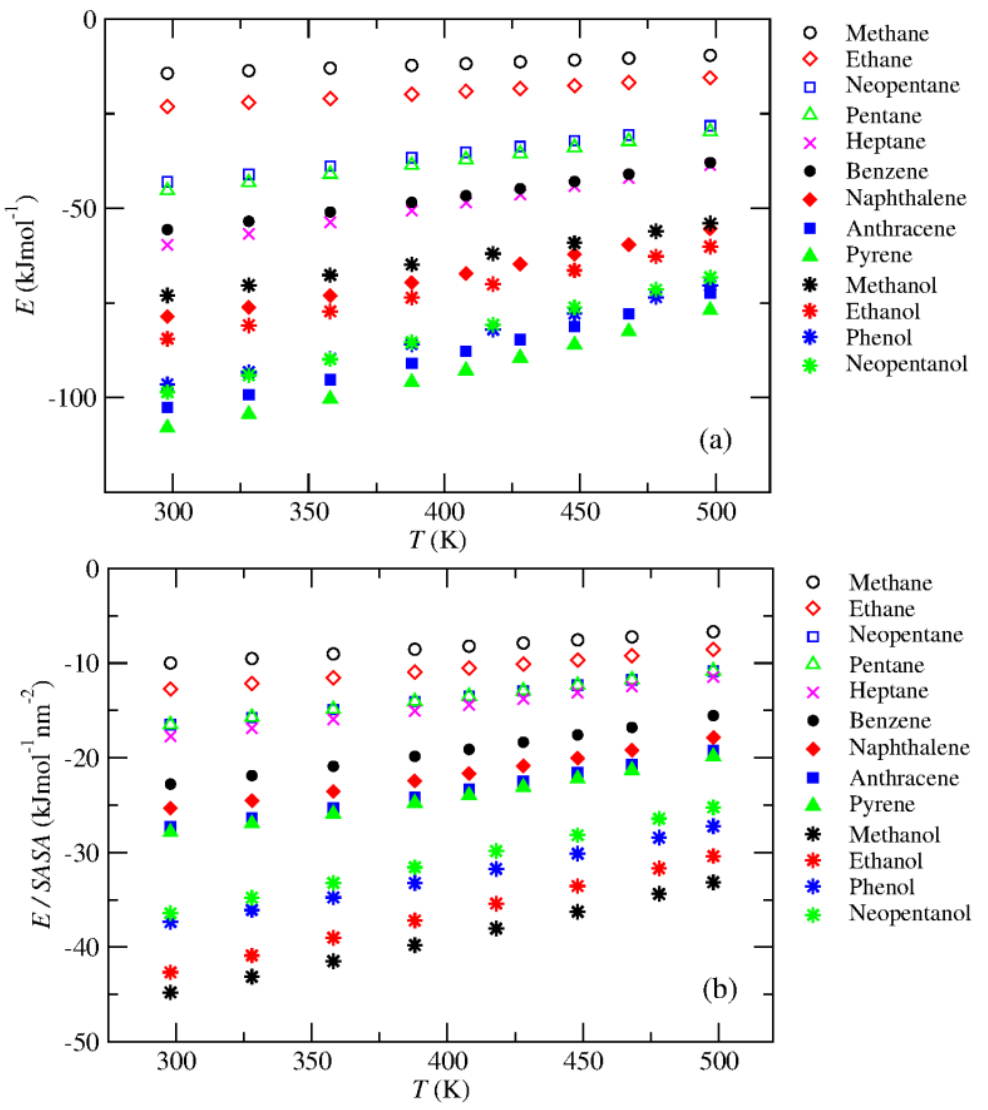

Figure S6 - Temperature dependence of the solute-water, (a) van der Waals and short range component of the Coulomb interaction energy, $E$, and (b) $E$ normalized by the SASA. 